CPHT-RR083.1108

\title{
Flux algebra, Bianchi identities and Freed-Witten anomalies in F-theory compactifications
}

\author{
G. Aldazabal ${ }^{a, c}$, P. G. Cámara ${ }^{b}$, J. A. Rosabal ${ }^{c}$ \\ ${ }^{a}$ Centro Atómico Bariloche, ${ }^{c}$ Instituto Balseiro (CNEA-UNC) and CONICET. \\ 8400 S.C. de Bariloche, Argentina. \\ ${ }^{b}$ Centre de Physique Théorique1, Ecole Polytechnique, \\ F-91128 Palaiseau, France.
}

\begin{abstract}
We discuss the structure of 4D gauged supergravity algebras corresponding to globally nongeometric compactifications of F-theory, admitting a local geometric description in terms of 10D supergravity. By starting with the well known algebra of gauge generators associated to nongeometric type IIB fluxes, we derive a full algebra containing all, closed RR and NSNS, geometric and non-geometric dual fluxes. We achieve this generalization by a systematic application of $S L(2, \mathbb{Z})$ duality transformations and by taking care of the spinorial structure of the fluxes. The resulting algebra encodes much information about the higher dimensional theory. In particular, tadpole equations and Bianchi identities are obtainable as Jacobi identities of the algebra. When a sector of magnetized $(p, q)$ 7-branes is included, certain closed axions are gauged by the $U(1)$ transformations on the branes. We indicate how the diagonal gauge generators of the branes can be incorporated into the full algebra, and show that Freed-Witten constraints and tadpole cancellation conditions for $(p, q)$ 7-branes can be described as Jacobi identities satisfied by the algebra mixing bulk and brane gauge generators.
\end{abstract}

\footnotetext{
${ }^{1}$ Unité mixte du CNRS, UMR 7644.
} 


\section{Contents}

1 Introduction $\quad 1$

2 Basics

2.1 Type IIB compactifications with non-geometric fluxes . . . . . . . . . 5

2.2 Non-geometric compactification of F-theory . . . . . . . . . . . . . 10

2.3 A non-geometric limit for F-theory on K3 . . . . . . . . . . . . . . . 14

3 Flux algebra from $S L(2, \mathbb{Z})^{7}$ dualities $\quad 18$

3.1 Flux algebra for $F_{3}$ and Q-fluxes . . . . . . . . . . . . 18

3.2 Inclusion of $H_{3}$ and $P$-fluxes . . . . . . . . . . . . . . . . . 22

4 Brane gaugings and Freed-Witten anomalies 25

4.1 A simple example . . . . . . . . . . . . . . . . . 26

4.2 Freed-Witten conditions and gaugings for magnetized D9-branes . . . . . . 28

4.3 Gaugings and Freed-Witten conditions for F-theory 7-branes . . . . . . . . 30

5 Comparison with gauged supergravity actions $\quad 34$

$5.1 \mathcal{N}=4$ gauged supergravity . . . . . . . . . . . . . . 34

$5.2 \mathcal{N}=1$ structure and D3-brane sector . . . . . . . . . . . 37

6 Summary and Outlook

A Background fluxes

\section{Introduction}

The presence of a variety of $p$-forms in ten dimensional superstring theories opens up the possibility of considering compactifications to lower dimensions where some background fluxes are turned on for their corresponding field strengths. In recent years these flux compactifications [1] (which include the so-called geometric fluxes, deforming the internal compactification manifold), have been studied from different perspectives. A particularly 
appealing consequence is that fields - which would be moduli in the absence of fluxes acquire a potential [2] and, thus, vacuum degeneracy can be lifted. This is particularly important for understanding the breaking of supersymmetry.

The effective four dimensional field theories resulting from superstring flux compactifications are described by gauged supergravities, namely, deformations of ordinary abelian supergravity theories where the deformation parameters (gaugings) correspond to the quantized fluxes. Matter fields become charged with respect to gauge fields which, generically, are non-abelian. A nice example of this situation is provided by Scherk-Schwarz compactifications [3] on twisted tori, where the isometries of the compact manifold become structure constants of the four dimensional gauge fields.

However, the connection between a string compactification and the gauged supergravity effective theory is quite subtle. For instance, it is known that orientifold compactifications of type IIA and type IIB 10D supergravity actions, in the presence of the corresponding background fluxes, lead to different superpotentials. Since both superstrings theories are connected through mirror symmetry, it was suggested in [4, 5] that new fluxes should be included in order for the full superpotentials to match. Similarly, more fluxes can be suggested by invoking type IIB S-duality, M-theory or heterotic/type I S-duality [5]. Hence, the resulting four dimensional gauged supergravity theory incorporates information about the stringy aspects of the starting configuration. Generically, it is not the reduction of a ten dimensional effective supergravity action.

Fluxes are strongly constrained by consistency conditions like Bianchi identities or tadpole cancellation equations. Part of such constraints can be derived from the ten dimensional effective supergravity. However, other necessary constraints, related to quantities encoding stringy aspects of the starting configuration, must be inferred by using string duality arguments. This is a particularly relevant issue when the study of moduli stabilization is addressed. Certain candidates for scalar potential minima could be actually forbidden by these constraints.

In this paper we take the orientifold framework of Ref. [5]. There, type IIB orientifold compactifications plus a $\mathbb{Z}_{2} \times \mathbb{Z}_{2}$ projection ensuring tori factorization is considered. In particular it is shown that $2^{7}$ dual fluxes are needed to ensure duality invariance and, 
moreover, these are arranged into spinor representations of $S L(2, \mathbb{Z})^{7}$. We focus on $2^{6}$ of these fluxes and argue that they describe globally non-geometric compactifications of F-theory, which admit a geometric local description in terms of 10D supergravity. Hence, in Section 2, we generalize the construction of [4] to allow for type IIB $S L(2, \mathbb{Z})$ transformations, apart from T-dualities, in the transition functions which glue different local patches. The canonical example is given by F-theory compactification on a K3 [6], where some of the 7-branes have been traded by closed string fluxes, leading to an axiondilaton which is not neutral under global monodromies. The resulting compact space is generically an U-fold [7, 8] (see also [9]).

Recently, the application of F-theory to model building has been explored [10, 11, 12, 13, 14, 15, showing interesting features, such as top Yukawa couplings of the order of the gauge coupling constant or the presence of exceptional gauge groups, suitable for GUT model building. On the other side, globally non-geometric compactifications may lead to moduli stabilization 1 [4, 5], or induce supersymmetric mass terms ( $\mu$-terms) in the worldvolume of D3 and D7-branes [16, 17, 18, 19, 20, 21]. Remarkably, supersymmetric Minkowski vacua with all closed string moduli stabilized were found in [22, 23] at the level of the 4D effective supergravity.

The aim of this paper is to provide the necessary tools for combining both setups, Ftheory configurations with exotic 7-branes and globally non-geometric compactifications, in a consistent manner. For that, we exploit the connection of string compactifications to gaugings in the four dimensional theory, and propose a systematic way to study the constraints that background fluxes must obey.

In a general compactification of F-theory there are three possible origins of four dimensional vector fields which can be gauged:

1. Kaluza-Klein vectors, arising from dimensional reduction of the metric and the NSNS/RR forms.

2. 7-brane gauge symmetries, arising at codimension two loci where the fiber, para-

\footnotetext{
${ }^{1}$ Although this stabilization manifests perturbatively in the four dimensional effective theory, we prefer not to use the term perturbative, since in our case the ten dimensional uplift is generically nonperturbative.
} 
metrizing the axion-dilaton dependence, degenerates into an ADE singularity. At points of the moduli space admitting an orbifold description, some of these vectors correspond to twisted states.

3. 3-brane gauge symmetries, completely localized along the compact directions.

In this work we analyze the gauged supergravity algebras associated to the first two classes of vector fields, and comment also on the constraints arising from 3-branes, when the latter sector is trivial. Our starting point is the algebra satisfied by a small set of gauge generators whose ten dimensional origin is well understood. A further systematic application of $S L(2, \mathbb{Z})^{7}$ transformations then leads to a complete algebra for Kaluza-Klein vectors, where the full set of fluxes is generated as structure constants. Jacobi identities satisfied by gauge generators lead to constraints on the allowed fluxes. Interestingly enough, from this four dimensional point of view, RR tadpole cancellation requirements and Bianchi identities appear at the same footing. We derive the tadpole cancellation requirements for $(p, q)$ 7-branes and show that some Jacobi identities impose stronger restrictions than other previously considered in the literature. This study is performed in Section 3 ,

In Section 4 we address the inclusion of the 7-brane sector. In principle, chiral configurations of branes can be described in the gauging formalism. One way is to start from e.g. type I with D9 branes, and then magnetize them. The magnetization simply amounts to introducing some worldvolume fluxes, which can be described as gaugings. Due to Chern-Simons couplings on the volume of the branes, sourced by the presence of magnetic fields, certain RR axionic scalars shift under D-brane $U(1)$ gauge symmetries.

From different points of view, it has been put forward that some D-brane configurations are not allowed for when bulk fluxes are switched on. The allowed brane configurations must satisfy some non-trivial generalized Freed-Witten [24] constraints, indicating a sort of mutual consistency between open and closed string fluxes. For instance, in [25] it was proposed to understand Freed-Witten constraints as the requirements needed to ensure invariance of the effective superpotential under shifts of the $U(1)$ gauged axionic scalars. Here we propose to study this interrelation between open and closed string fluxes, from 
the point of view of the complete algebra describing both, bulk and worldvolume gaugings. The idea is that Freed-Witten constraints could arise as Jacobi identities for such algebra. We find that this is indeed the case, at least for the subset of fluxes that we are able to manage. We derive in this way the Freed-Witten conditions that $(p, q) 7$-branes have to satisfy in non-geometric compactifications of F-theory.

In Section 5 we discuss the connection between $\mathcal{N}=4$ supergravity actions and $\mathcal{N}=1$ superstring compactifications. We expect our string compactification to lead in four dimensions to a truncation of an $\mathcal{N}=4$ gauged supergravity theory, due to the presence of fluxes and the orientifold projection. Indeed, we show that a precise dictionary between the $\mathcal{N}=4$ orbifold truncated gauged algebra and the one derived in Section 3 can be established. Jacobi identities do match quadratic constraints on the possible gaugings, required for consistency of gauged supergravity theories.

Finally, Section 6 contains some conclusions and an outlook. We collect in the Appendix different tables containing fluxes and their transformation properties.

\section{Basics}

\subsection{Type IIB compactifications with non-geometric fluxes}

We consider type IIB orientifold compactifications on $T^{6} /\left[\Omega_{P}(-1)^{F_{L}} \sigma\right]$, where $\Omega_{P}$ is the worldsheet parity operator, $(-1)^{F_{L}}$ is the space-time fermionic number for the left-movers and $\sigma$ is an involution acting on the Kähler form and the holomorphic 3-form, respectively as $\sigma(J)=J$ and $\sigma(\Omega)=-\Omega$. In general there can be O3-planes spanning the space-time directions.

For simplicity we also assume an underlying $\mathbb{Z}_{2} \times \mathbb{Z}_{2}$ symmetry, so the 6-torus is factorized. Prior to the inclusion of background fluxes and stringy monodromies, the metric of the internal torus reads

$$
d s^{2}=\sum_{k=1}^{3} \frac{\operatorname{Re} T_{k}}{\operatorname{Re} U_{k}}\left|d x^{k}+i U_{k} d x^{k+3}\right|^{2},
$$

where $U_{k}$ are the complex structure moduli and $T_{k}$ are the Kähler moduli, defined in 
terms of the RR 4-form and the Kähler 2-form

$$
\mathcal{J}_{c} \equiv C_{4}+\frac{i}{2} e^{-\phi} J \wedge J=i \sum_{i=1}^{3} T_{i} \tilde{\omega}_{i}
$$

Here, $\tilde{\omega}_{i}$ denotes a basis of invariant 4 -forms.

The axion-dilaton modulus is given by

$$
S=e^{-\phi}+i C_{0}
$$

with $C_{0}$ the RR 0 -form. In the presence of 7 -branes, it is a holomorphic function of the internal coordinates. The four dimensional field, $S$, is then defined as the constant mode of the ten dimensional fields.

The seven moduli of the compactification, $\left(S, T_{k}, U_{k}\right), k=1,2,3$, span a Kähler manifold with potential

$$
K=-\log (S+\bar{S})-\sum_{k=1}^{3} \log \left[\left(T_{k}+\bar{T}_{k}\right)\left(U_{k}+\bar{U}_{k}\right)\right]
$$

The orientifold involution allows for two types of BPS object:2: D3-branes and $(p, q)$ $7{ }_{k}$-branes, with RR charge $p$ and NSNS charge $q$. The first ones span the four space-time dimensions, whereas the latter wrap also a $(2,2)$-cycle, $\left[\tilde{\omega}_{k}\right]$, in the internal 6-torus.

Consistently with the orientifold involution, it is possible to consider in addition nontrivial backgrounds for the RR and NSNS 3-forms, $F_{3}$ and $H_{3}$, satisfying a BPS-like condition [26]. The fluxes induce a deformation on the moduli space which, from the four dimensional perspective, can be effectively parameterized in terms of the superpotential $[2]$

$$
W=\int\left(F_{3}-i S H_{3}\right) \wedge \Omega
$$

Due to the Chern-Simons coupling

$$
\int C_{4} \wedge H_{3} \wedge F_{3}
$$

present in the type IIB supergravity action, the fluxes induce a smeared charge of D3brane which, for consistency, must be cancelled by the effect of localized sources. This

\footnotetext{
${ }^{2}$ Strictly speaking, also D9-branes with suitably magnetization are allowed in certain regions of the moduli space. Here, however, we only focus on 3 and 7-branes.
} 
leads to the condition,

$$
\frac{1}{2 \cdot 3 !} \tilde{F}^{m n p} H_{m n p}=N_{D 3 / O 3}
$$

where $N_{D 3 / O 3}$ denotes the total D3-brane charge associated with localized objects and,

$$
\tilde{F}^{i j k} \equiv \frac{1}{3 !} \epsilon^{i j k o p q} F_{o p q} .
$$

Since D3-branes are related to 3-form fluxes through brane/flux transmutation [27, 28, the D3 tadpole cancellation condition can be interpreted as a charge conservation requirement, rather than a static constraint.

In [4, 5] another set of "flux" deformations was considered in the effective theory in order to restore mirror symmetry between type IIB and type IIA orientifold compactifications in the presence of non-vanishing 3 -form fluxes. The idea is that T-duality acts on the NSNS 3-form flux through the chain

$$
H_{m n p} \stackrel{\mathrm{T}_{m}}{\longleftrightarrow}-\omega_{n p}^{m} \stackrel{\mathrm{T}_{n}}{\longleftrightarrow}-Q_{p}^{m n} \stackrel{\mathrm{T}_{p}}{\longleftrightarrow} R^{m n p}
$$

Since T-duality exchanges Kaluza-Klein and winding modes, the stringy nature of these deformations increases as we move to the right in this chain and, in particular, ten dimensional supergravity starts failing to be a good description. Thus, $\omega$-fluxes are geometric fluxes, describing compactifications on twisted tori with structure constants $\omega_{j k}^{i}$, as discussed below. Q-fluxes parameterize compactifications on T-folds, where T-duality is used, together with diffeomorphisms, to glue different local patches of the manifold together. These are globally non-geometric constructions, but locally geometric, sometimes related to the effective "geometry" of asymmetric orbifolds [29, 30, 31, 32]. Finally, Rfluxes correspond to highly non-geometric compactifications, which do not even admit a local geometric description.

The orientifold projection we have chosen projects all the geometric and R-fluxes out. Only, 24 components of $Q_{k}^{i j}$ survive the projection, as summarized in the Appendix. These can be thought as the structure constants of a gauge algebra [4, 5]

$$
\left[X^{i}, X^{j}\right]=Q_{p}^{i j} X^{p}
$$

where $X^{i}$ are the gauge group generators associated to vector fields which, in the T-dual type I picture, result from dimensional reduction of the metric, as we will see in a moment. 
In particular, Q-fluxes must satisfy the Jacobi identity of the algebra

$$
Q_{p}^{[m n} Q_{t}^{r] p}=0
$$

To gain a more intuitive understanding of this algebraic structure, a brief parenthesis describing the T-dual type I compactification with O9-planes is worthwhile. Performing six T-dualities along the internal 6-torus, Q-fluxes are mapped into geometric fluxes

$$
Q_{p}^{q r} \rightarrow \omega_{q r}^{p}
$$

These have a clear interpretation from the point of view of the compactified theory. In an ordinary Kaluza-Klein compactification on a torus, the different fields only depend on the space-time coordinates and we must deal with exact differentials $d x^{i}$ for the internal tori. However, a Scherk-Schwarz [3] like compactification can be achieved by allowing for a controlled dependence of the fields on the internal coordinates $x^{a}(a=1, \ldots 6)$. The reduction is realized in terms of an expansion around a non-trivial metric described by new twisted tori vielbeins $\eta^{c}=U_{p}^{c}(x) d x^{p}$. The functions $U_{p}^{c}(x)$, encoding the dependence on internal coordinates, are not completely arbitrary. They must be such that the vielbein is a globally defined basis of 1 -forms such that

$$
d \eta^{c}=-\frac{1}{2} \omega_{a b}^{c} \eta^{a} \wedge \eta^{b}
$$

with $\omega_{a b}^{c}$ constant.

For the vector bosons, $A_{\mu}^{a} \equiv g_{\mu}{ }^{a}$, built up from the ten dimensional metric $g_{m n}$, the gauge transformations read [33]

$$
\delta g_{\mu}{ }^{c}=\partial_{\mu} \lambda^{c}-\omega_{a b}^{c} \lambda^{a} g_{\mu}{ }^{b}
$$

where $\lambda^{c}$ are the group parameters. Thus, the metric fluxes $\omega_{a b}^{c}$ correspond to the gauge group structure constants. By introducing the gauge group generators $Z_{p}$

$$
A_{\mu}=g_{\mu}^{p} Z_{p}
$$

we find

$$
\left[Z_{a}, Z_{b}\right]=\omega_{a b}^{p} Z_{p}
$$


with $a=1, \ldots 6$. This is not other but the T-dual algebra to eq.(2.10). The Jacobi identities of the algebra, for each $Z_{l}$, lead to the constraints,

$$
\omega_{[a b}^{p} \omega_{c] p}^{l}=0
$$

which correspond to the Bianchi identities derived from (2.13) and which are dual of (2.11). In Section 4 we will find convenient to use this type I description to elaborate on the Freed-Witten constraints which arise from the flux algebra.

Let us now return to the type IIB picture with Q-fluxes. From the ten dimensional point of view, the $Q_{k}^{i j}$ fluxes lead to local supergravity solutions which interpolate between different standard $\mathcal{N}=1$ orientifold involutions [34] (see also [35, 36]). Qualitatively, these are characterized by a non-constant holomorphic axion-dilaton which transforms under global monodromies of the manifold, with $S\left(x^{k}+1\right)$ being related to $S\left(x^{k}\right)$ through T-duality transformations.

In many senses, the background locally resembles a standard type IIB compactification with 3-form fluxes. However, the B-field contains an extra dependence on the dilaton,

$$
B_{2} \simeq e^{\phi\left(x^{k}\right)} Q_{k}^{a b} x^{k} d x^{a} \wedge d x^{b}+\ldots
$$

Thus, due to the term in the Wess-Zumino action

$$
\int C_{6} \wedge B_{2}
$$

a D7-brane wrapping a 4-cycle which contains the 2-cycle $\left[d x^{a} \wedge d x^{b}\right]$, experiences a solitonic D5-brane charge in the worldvolume which, due to the non-linear dependence of the dilaton on $x^{k}$, cannot be absorbed by means of a gauge transformation of the Bfield. In order to perform the identification $x^{k} \sim x^{k}+1$, a stringy monodromy (two T-duality transformations along $x^{a}$ and $x^{b}$ ) is required, so that the change in the dilaton is compensated.

In the four dimensional theory, the deformation on the moduli space induced by these stringy monodromies can be parameterized in terms of the superpotential [5]

$$
W=\int\left(F_{3}-i S H_{3}+Q \mathcal{J}_{c}\right) \wedge \Omega
$$


where we have defined the $Q$-product as

$$
(Q X)_{p m_{1} \ldots m_{p-2}}=\frac{1}{2} Q_{[p}^{a b} X_{\left.m_{1} \ldots m_{p-2}\right] a b} .
$$

In addition, Q-fluxes induce a D7-brane charge

$$
-\int C_{8} \wedge Q F_{3}
$$

which must be cancelled by the effect of localized sources (D7-branes and/or O7-planes). By analogy with the D3-brane case, it is then natural to conjecture that Q-fluxes are related to D7-branes through (non-)geometric transitions.

At first sight, it may seem surprising that the four dimensional effective theory captures part of the stringy nature of the original compactification. Indeed, it is perhaps more appropriate to think of the four dimensional effective supergravity as being the low energy limit of a ten dimensional string setup, rather than a ten dimensional supergravity background. States of the higher dimensional theory which are protected by holomorphicity are expected to be captured by the superpotential in the four dimensional effective supergravity. Up to what extent the superpotential (2.20) gives a complete description of the light modes in compactifications with non-vanishing Q-fluxes is, however, still an open question.

\section{$2.2 \quad$ Non-geometric compactification of F-theory}

Type IIB string theory is conjectured to be invariant under S-duality. This is reflected in a $S L(2, \mathbb{R})$ self-duality of the type IIB supergravity equations of motion, broken to $S L(2, \mathbb{Z})$ at the quantum level. Moreover, whereas in ten dimensions S-duality is a weak-strong coupling duality, leading to the exchange of winding modes and E1-instanton effects, in four dimensions it manifests as weak-weak electric-magnetic duality. This is the idea behind the adiabatic argument of Vafa-Witten [37, providing us with a prescription for building S-dual pairs of ten dimensional superstring compactifications. It is therefore natural to conjecture that the dynamics of some light modes, protected by holomorphicity, in strongly coupled compactifications of type IIB string theory, is partially captured by gaugings in the four dimensional effective supergravity. 
The complex axion-dilaton transforms under $S L(2, \mathbb{Z})$ as

$$
S \rightarrow \frac{k S-i \ell}{i m S+n}, \quad k n-\ell m=1, \quad k, \ell, m, n \in \mathbb{Z},
$$

whereas 3-form fluxes behave as a doublet

$$
\left(\begin{array}{l}
F_{3} \\
H_{3}
\end{array}\right) \rightarrow\left(\begin{array}{ll}
k & \ell \\
m & n
\end{array}\right)\left(\begin{array}{l}
F_{3} \\
H_{3}
\end{array}\right),
$$

and $C_{4}$ remains invariant. With these transformation rules at hand, we may easily check that the D3-brane tadpole cancellation condition, eq.(2.7), and the flux superpotential, eq.(2.5), are invariant under S-duality, up to Kähler transformations of the superpotential.

When D7-branes and/or $Q$-fluxes are also present, the $S L(2, \mathbb{Z})$ invariance of the theory becomes more subtle. The RR 8 -form, $C_{8}$, is one of the components of a $S L(2, \mathbb{Z})$ triplet of 8-forms, $\left(C_{8}, \tilde{C}_{8}, C_{8}^{\prime}\right)[38,39$, 40, 41]. Under $S \rightarrow 1 / S$ the elements of the triplet transform as

$$
\begin{aligned}
& C_{8} \rightarrow-\tilde{C}_{8}, \\
& \tilde{C}_{8} \rightarrow-C_{8}, \\
& C_{8}^{\prime} \rightarrow-C_{8}^{\prime} .
\end{aligned}
$$

There are 7-branes coupling electrically to all these forms, with charges that we denote as $p^{2}, q^{2}$ and $r$, respectively. These can be arranged into a $S L(2, \mathbb{Z})$-invariant traceless matrix of charges

$$
\mathcal{N}_{7} \equiv\left(\begin{array}{cc}
r / 2 & p^{2} \\
-q^{2} & -r / 2
\end{array}\right) \text {. }
$$

The charge matrix $\mathcal{N}_{7}$ determines the $S L(2, \mathbb{Z})$ monodromy suffered by the axion-dilaton when going in a closed path around the 7-brane [41]

$$
S \rightarrow\left[\cos \left(n_{7}^{1 / 2}\right) I+\frac{\sin \left(n_{7}^{1 / 2}\right)}{n_{7}^{1 / 2}} \mathcal{N}_{7}\right] S,
$$

with $n_{7} \equiv \operatorname{det} \mathcal{N}_{7}$ and $I$ the $2 \times 2$ identity matrix.

Physical 7-branes transform as non-linear doublets of $S L(2, \mathbb{Z})$ [41, so that there are only two independent entries in $\mathcal{N}_{7}$. Different $S L(2, \mathbb{Z})$ orbits are characterized by different values of $n_{7}$. 
In the uplift to F-theory, $(p, q)$ 7-branes are associated to codimension two loci where the elliptic fiber, which parameterizes the axion-dilaton dependence on the compact coordinates, degenerates into an ADE singularity. Therefore, F-theory $(p, q)$ 7-branes lay in the same orbit as the D7-brane, characterized by the condition $n_{7}=0$ [41].

We denote the total charge associated to $(p, q) 7_{k}$-branes by the matrix

$$
\left(\mathcal{N}_{7_{k}}\right)_{\text {total }}=\sum_{I}\left(\begin{array}{cc}
p_{k}^{I} q_{k}^{I} & \left(p_{k}^{I}\right)^{2} \\
-\left(q_{k}^{I}\right)^{2} & -p_{k}^{I} q_{k}^{I}
\end{array}\right)
$$

where the sum extends over all $7_{k}$-branes present in the model. In general, $\operatorname{det}\left(\mathcal{N}_{7_{k}}\right)_{\text {total }} \neq$ 0 , and the configuration cannot be rotated by a $S L(2, \mathbb{Z})$ transformation into a setup with only D7-branes. The configuration is, thus, intrinsically non-perturbative. On the other hand, objects with $n_{7} \neq 0$ can be seen as non-perturbative bound states of $(p, q)$ 7-branes. Notice that O7-planes are also implicitly included in (2.30), as they are the weak coupling limit of compound objects made up of several non-perturbative $(p, q)$ 7-branes [6, 41].

From eqs.(2.20) and (2.22) it is evident that Q-fluxes must transform in a non-trivial way to keep the $S L(2, \mathbb{Z})$ invariance of the theory. In [5], it was conjectured that such fluxes belong to a linear doublet of $S L(2, \mathbb{Z})$, so a new set of flux parameters, $P_{k}^{i j}$, was considered

$$
\left(\begin{array}{l}
Q \\
P
\end{array}\right) \rightarrow\left(\begin{array}{ll}
k & \ell \\
m & n
\end{array}\right)\left(\begin{array}{l}
Q \\
P
\end{array}\right)
$$

We summarize in the Appendix the 24 components of $P_{k}^{i j}$ which are compatible with the $\mathbb{Z}_{2} \times \mathbb{Z}_{2}$ symmetry.

The effective superpotential and Chern-Simons couplings, eqs.(2.20) and (2.22), must be extended accordingly to the expressions

$$
W=\int\left[\left(F_{3}-i S H_{3}\right)+(Q-i S P) \mathcal{J}_{c}\right] \wedge \Omega
$$

and

$$
\int-C_{8} \wedge Q F_{3}+\tilde{C}_{8} \wedge P H_{3}+C_{8}^{\prime} \wedge\left(Q H_{3}+P F_{3}\right),
$$

Therefore, $P$ and $Q$-fluxes naturally source the tadpole cancellation requirements associ- 
ated to the total $(p, q) 7_{k}$-brane charge

$$
\begin{aligned}
& \left(Q H_{3}+P F_{3}\right)_{k}=2 \sum_{I} p_{k}^{I} q_{k}^{I}, \\
& \left(Q F_{3}\right)_{k}=\sum_{I}\left(p_{k}^{I}\right)^{2} \\
& \left(P H_{3}\right)_{k}=\sum_{I}\left(q_{k}^{I}\right)^{2}
\end{aligned}
$$

where we have defined the P-product in a similar way to the Q-product, eq.(2.21), and we have expanded the 1.h.s. in a basis of 2-forms, $\omega_{k}, k=1,2,3$, dual to $\tilde{\omega}_{k}$.

In a similar fashion to what occurs with the rest of the flux parameters, it is natural to expect that P-fluxes also must satisfy a set of Bianchi identities. The ansatz for some of these constraints was already presented in [5], based on symmetry arguments. In Section 3, however, we derive in a more systematic and precise way the full set of constraints that the P-fluxes must satisfy, finding some differences with respect to [5].

Since the $S L(2, \mathbb{Z})$ symmetry, relating $\mathrm{P}$ and Q-fluxes, is an explicit symmetry of the type IIB supergravity equations of motion, we also expect the $P$-flux parameters to correspond to compactifications which admit a ten dimensional local supergravity description. Indeed, acting with $S L(2, \mathbb{Z})$ in each local patch of a compactification with non-vanishing Q-flux, we observe that (2.18) is translated into a dilaton dependence for the RR 2-form potential, whenever a non-vanishing $P_{k}^{i j}$ flux is turned on

$$
C_{2} \simeq e^{\phi\left(x^{k}\right)} P_{k}^{a b} x^{k} d x^{a} \wedge d x^{b}+\ldots
$$

Hence, for instance, a (0,1) 7-brane wrapping a 4-cycle containing $\left[d x^{a} \wedge d x^{b}\right]$, will experience a solitonic NS5-brane charge depending on its position in $x^{k}$, induced by the coupling in the Wess-Zumino action

$$
\int B_{6} \wedge C_{2}
$$

The stringy monodromy gluing $x^{k} \simeq x^{k}+1$, in this case involves an S-duality transformation followed by two T-dualities along $x^{a}$ and $x^{b}$ and a further S-duality. Since $\mathrm{S}$ and T-dualities do not commute, the total monodromy is not equivalent to just performing two T-dualities. 
For vanishing fluxes, holomorphicity and neutrality under monodromies are enough to determine the profile of $S$ in terms of the modular invariant holomorphic $j$-function [6]. In the presence of $\mathrm{Q}$ and/or P-fluxes, however, the holomorphic axion-dilaton, $S$, is no longer monodromy neutral. In the next section, we present some simple explicit examples which will serve to clarify the above discussion.

\subsection{A non-geometric limit for F-theory on K3}

Let us consider Sen's construction of F-theory compactified on a elliptically fibered K3 [6]. The later is given by the Weierstrass curve

$$
\hat{y}^{2}=\hat{x}^{3}+P(z) \hat{x}+Q(z)
$$

where $z=x^{3}+i U_{3} x^{6}$ is the coordinate of the $C P^{1}$ base, $\hat{x}$ and $\hat{y}$ the coordinates of the fibre, and $P^{3}+Q^{2}$ is a polynomial of order 24 , whose zeros correspond to the locations of the 7 -branes transverse to the $C P^{1}$. Global consistency requires 24 D7-branes. The axion-dilaton is given in terms of the modular parameter of the fibre

$$
S(z)=-i j^{-1}\left(\frac{P^{3}(z)}{P^{3}(z)+Q^{2}(z)}\right),
$$

with $j$ the modular invariant $j$-function. In the limit on which $P^{3}(z) \propto Q^{2}(z)$, the axiondilaton becomes constant and the $C P^{1}$ degenerates into a $T^{2} / \mathbb{Z}_{2}$ orbifold. Each of the four singularities, $\left(x^{3}, x^{6}\right)=\{(0,0),(1 / 2,0),(0,1 / 2),(1 / 2,1 / 2)\}$, corresponds to a bound state of $(p, q)$ 7-branes, which in the weak coupling limit manifests as 4 D7-branes, each one with charge +1 , and a single O7-plane, with charge -4 . The four dimensional theory

resulting from further compactification in $T_{\left[x^{1} x^{4}\right]}^{2} \times T_{\left[x^{2} x^{5}\right]}^{2}$ is $\mathcal{N}=4$ supersymmetric. More generically, some of the 7 -branes may be magnetized, leading to $\mathcal{N}=2,1,0$ supersymmetry in four dimensions.

Being slightly more general, we can perform an $S L(2, \mathbb{Z})$ rotation of the above system by the matrix

$$
\Lambda_{(\mathfrak{p}, \mathfrak{q})}=\left(\begin{array}{cc}
\mathfrak{p} & \mathfrak{a} \\
-\mathfrak{q} & \mathfrak{b}
\end{array}\right)
$$


with $(\mathfrak{p}, \mathfrak{q})$ two mutually prime integers, and $(\mathfrak{a}, \mathfrak{b})$ one of the infinite solutions of the Bézout's identity

$$
\mathfrak{p} \mathfrak{b}+\mathfrak{q a}=1
$$

After performing this rotation, the 16 D7-branes become $16(\mathfrak{p}, \mathfrak{q})$ 7-branes, whereas each of the O7-planes becomes a different bound state of $(p, q)$ 7-branes, with total charge $-4(\mathfrak{p}, \mathfrak{q})$.

Based on the tadpole cancellation conditions, eqs.(2.34)-(2.36), we expect $(p, q) 7$ branes to be related to $\mathrm{Q}$ and P-fluxes by non-geometric transitions. We would like to consider here the case on which $N^{2} / 4$ of the $4(\mathfrak{p}, \mathfrak{q}) 7$-branes at each singularity, with $N=0,2,4$, have been traded by fluxes. The resulting closed string background must satisfy the 7-brane charge conservation conditions, given by eqs.(2.34)-(2.36). A possible choice of the flux parameters satisfying these requirements is given by,

$$
\begin{gathered}
Q_{6}^{42}=-Q_{6}^{15}=-F_{423}=F_{153}=\mathfrak{p} N \\
P_{6}^{15}=-P_{6}^{42}=-H_{153}=H_{423}=\mathfrak{q} N
\end{gathered}
$$

Indeed, plugging (2.43) into (2.32) it is not difficult to check that there is a $\mathcal{N}=2$ supersymmetric Minkowski vacuum with $U_{3}=T_{3}$ and $U_{1}=U_{2}$. Interestingly enough, performing an inverse $S L(2, \mathbb{Z})$ transformation, $\Lambda_{(\mathfrak{p}, \mathfrak{q})}^{-1}$, followed by 4 T-dualities along $T_{\left[x^{1} x^{4}\right]}^{2} \times T_{\left[x^{2} x^{5}\right]}^{2}$, the background (2.43) is dual to a type IIB toroidal compactification with standard 3-form fluxes, whose ten dimensional solution is well known [42, 43, 26]. Hence, in the same spirit than [34, we can perform the above chain of dualities, starting with the dual background, to obtain the ten dimensional supergravity solution associated to the flux parameters (2.43).

The initial type IIB configuration with 3 -form fluxes is the same than the one considered in [34]. Let us concentrate first in the case where $\Lambda_{(\mathfrak{p}, \mathfrak{q})}=\mathbb{I}$. Performing 4 T-dualities and a redefinition of the moduli parameters, the resulting ten dimensional solution can 
be written as 3 ,

$$
\begin{aligned}
d s^{2} & =Z^{-1 / 2} d s_{\mathbb{R}^{1,3}}^{2}+Z^{1 / 2}\left[\sum_{k=1,2} \frac{t_{3} e^{\phi} t_{k}}{\operatorname{Re} U}\left|d x^{k}+i U d x^{k+3}\right|^{2}+\frac{Z^{1 / 2}}{g_{s} t_{3}}\left|d x^{3}+i T_{3} d x^{6}\right|^{2}\right] \\
C_{2} & =N x^{3}\left(d x^{1} \wedge d x^{5}-d x^{4} \wedge d x^{2}\right) \\
B_{2} & =N e^{\phi} t_{3} x^{6}\left(d x^{1} \wedge d x^{5}-d x^{4} \wedge d x^{2}\right) \\
F_{1} & =*_{T_{\left[x^{3} x^{6}\right]}^{2}} d e^{-\phi} \\
e^{\phi} & =\frac{t_{3}^{-1}}{\left(N x^{6}\right)^{2}+t_{1} t_{2} Z} \\
F_{5} & =C_{2} \wedge H_{3}+F_{3} \wedge B_{2}-\frac{1}{2} F_{1} \wedge B_{2} \wedge B_{2}-C_{0} \wedge H_{3} \wedge B_{2}
\end{aligned}
$$

where $U, g_{s}, t_{1,2}$ and $t_{3} \equiv \operatorname{Re} T_{3}$ are constant modular parameters, and $Z\left(x^{3}, x^{6}\right)$, with is the warp factor in the dual type IIB configuration with 3 -form fluxes. In terms of these parameters, $Z\left(x^{3}, x^{6}\right)$ satisfies the equation

$$
-\tilde{\nabla}_{T_{\left[x^{3} x^{6}\right]}^{2} Z}^{2} Z=\frac{2 g_{s} N^{2}}{t_{1} t_{2} t_{3}}-\frac{N^{2}}{2} \sum_{m, n=0,1 / 2} \delta\left(x^{3}-m, x^{6}-n\right)
$$

From eqs. (2.46) and (2.47), we observe that in order to compute $S$ we have to solve this equation. From an open string dual perspective, this is equivalent to compute the gauge threshold corrections of a probe D3-brane [44].

Making use of the results of [44] (see also [45]) we obtain

$$
Z=1-\frac{N^{2}}{t_{1} t_{2} t_{3}}\left[\frac{(\operatorname{Im} z)^{2}}{t_{3}}-\frac{1}{8 \pi}\left|\vartheta_{1}\left(2 z,-i T_{3}\right)\right|^{2}\right]
$$

which plugged into (2.44)-(2.47) results in,

$$
\begin{aligned}
S & =t_{1} t_{2} t_{3}+\frac{N^{2}}{8 \pi} \log \vartheta_{1}\left(2 z,-i T_{3}\right), \\
C_{2} & =N x^{3}\left(d x^{1} \wedge d x^{5}-d x^{4} \wedge d x^{2}\right), \\
B_{2} & =\frac{N t_{3} x^{6}}{t_{1} t_{2} t_{3}+\frac{N^{2}}{8 \pi} \log \left|\vartheta_{1}\left(2 z,-i T_{3}\right)\right|^{2}}\left(d x^{1} \wedge d x^{5}-d x^{4} \wedge d x^{2}\right) .
\end{aligned}
$$

The moduli space of the resulting non-geometric compactification is given by the positions of the $24-N^{2}$ D7-branes. Indeed, under four T-dualities these are mapped to D3-branes,

\footnotetext{
${ }^{3}$ We thank F. Marchesano for sharing with us some of his notes where this setup was also considered.
} 
which do not receive supersymmetric mass terms from 3-form fluxes, and therefore can move freely on the bulk.

When one of the D7-branes at the origin moves along the $x^{6}$ direction, it develops a charge of D5-brane in its worldvolume, induced by the Chern-Simons coupling of $C_{6}$ to $B_{2}$, the latter given in eq.(2.52), in the worldvolume action. In order to identify $x^{6} \simeq x^{6}+1$, two T-dualities are needed in the corresponding transition function, as pointed out in [34].

Compactifications with non-vanishing Q-flux are often related, in special points of the moduli space, to asymmetric orbifolds [29, 30, 31, 32]. It is tempting to believe that, in the case at hand, where some of the D7-branes in the $T^{4} / \mathbb{Z}_{2}$ orbifold limit of F-theory on a K3 have been traded by fluxes, there is still a valid (asymmetric) orbifold description. If such is the case, the geometric moduli of the remaining D7-branes would parameterize the corresponding "blow-up" modes of the asymmetric orbifold. However, wether this is a valid description remains an open question.

To finish this section, let us consider now the general case, in which $\Lambda_{(\mathfrak{p}, \mathfrak{q})}$ is an arbitrary $S L(2, \mathbb{Z})$ rotation given in eq.(2.41) . It is simple to see that eqs.(2.50)-(2.52) are modified to

$$
\begin{aligned}
S & =\frac{8 \pi\left(\mathfrak{p} t_{1} t_{2} t_{3}-i \mathfrak{a}\right)+\mathfrak{p} N^{2} \log \vartheta_{1}\left(2 z,-i T_{3}\right)}{8 \pi(\mathfrak{b}-i \mathfrak{q})-i \mathfrak{q} N^{2} \log \vartheta_{1}\left(2 z,-i T_{3}\right)} \\
C_{2} & =N\left(\mathfrak{p} x^{3}+\mathfrak{a} \frac{t_{3} x^{6}}{t_{1} t_{2} t_{3}+\frac{N^{2}}{8 \pi} \log \left|\vartheta_{1}\left(2 z,-i T_{3}\right)\right|^{2}}\right)\left(d x^{1} \wedge d x^{5}-d x^{4} \wedge d x^{2}\right) \\
B_{2} & =N\left(-\mathfrak{q} x^{3}+\mathfrak{b} \frac{t_{3} x^{6}}{t_{1} t_{2} t_{3}+\frac{N^{2}}{8 \pi} \log \left|\vartheta_{1}\left(2 z,-i T_{3}\right)\right|^{2}}\right)\left(d x^{1} \wedge d x^{5}-d x^{4} \wedge d x^{2}\right)
\end{aligned}
$$

corresponding to the ten dimensional local realization of the flux parameters (2.43), whereas the metric and the RR 5 -form are independent of $\Lambda_{(\mathfrak{p}, \mathfrak{q})}$. Now there are $4-(N / 2)^{2}$ $(\mathfrak{p}, \mathfrak{q})$ 7-branes at each of the four singularities, which can move freely along $T_{\left[x^{3} x^{6}\right]}^{2}$. In this case, the Wess-Zumino action for $(p, q)$ 7-branes contains a term [46]

$$
p C_{6} \wedge B_{2}+q B_{6} \wedge C_{2}+\ldots
$$

so that $(\mathfrak{p}, \mathfrak{q})$ 7-branes develop a $(\mathfrak{p}, \mathfrak{q})$ 5-brane charge in its worldvolume depending on their position in $x^{6}$ and an $S L(2, \mathbb{Z})$ rotation by $\Lambda_{(\mathfrak{p}, \mathfrak{q})}^{-1}$, followed by two T-dualities and an 
inverse rotation by $\Lambda_{(\mathfrak{p}, \mathfrak{q})}$, have to be carried on in the transition functions gluing different patches, as it was advanced in the previous section.

In general, the axion-dilaton $S$, will also depend on the positions of the $16-N^{2} 7$ branes, as occurs in the flux-less case. Here, we have solved for the warping in the special point of the moduli space where the 7-branes are equally distributed on top of the four singularities. The expression we have found for the axion-dilaton, eq.(2.53), in general is not valid away from this special point. However, although $C_{2}$ and $B_{2}$ depend also on $S$ we expected that, to leading order, the dependence of these fields on $x^{6}$ is always $\mathcal{O}\left(x^{6}\right)$.

\section{$3 \quad$ Flux algebra from $S L(2, \mathbb{Z})^{7}$ dualities}

\subsection{Flux algebra for $F_{3}$ and Q-fluxes}

We proceed now to derive the full gauge algebra, starting from eq.(2.10), associated to Kaluza-Klein vectors in the class of non-geometric F-theory compactifications described in the previous section, and see what kind of information we can extract.

The global symmetry group associated to the moduli space of the factorized 6-torus is $S L(2, \mathbb{Z})^{7}$, where each of the $S L(2, \mathbb{Z})_{k}$ factors acts on a different modulus and consists of the generators

$$
\mathcal{S}_{k, 1}=\left(\begin{array}{ll}
1 & 1 \\
0 & 1
\end{array}\right), \quad \mathcal{S}_{k, 2}=\left(\begin{array}{cc}
0 & 1 \\
-1 & 0
\end{array}\right), \quad k=0, \ldots, 6 .
$$

The seven untwisted moduli can be grouped in the vector representation of $S L(2, \mathbb{Z})^{7}$

$$
\mathbb{T} \equiv \mathbf{7}=\left(S ; T_{1}, T_{2}, T_{3} ; U_{1}, U_{2}, U_{3}\right)
$$

whereas the flux parameters surviving the $\mathbb{Z}_{2} \times \mathbb{Z}_{2}$ projection transform in the spinorial representation, $\mathbb{G} \equiv \mathbf{1 2 8}[5]$. Labelling the components of $\mathbb{G}$ by the set of weights

$( \pm, \pm, \pm, \pm, \pm, \pm, \pm)$, where \pm stands for $\pm \frac{1}{2}$, under $\mathbb{T}_{k} \rightarrow 1 / \mathbb{T}_{k}$ the flux parameters transform as

$$
\mathcal{S}_{k, 2}\left(n_{0}, \ldots, n_{k}, \ldots, n_{6}\right)=\operatorname{sign}\left(n_{k}\right)\left(n_{0}, \ldots,-n_{k}, \ldots, n_{6}\right)
$$


Notice that 64 of these flux parameters correspond to the $H_{3}, F_{3}, Q$ and $P$-fluxes discussed in the previous section, describing F-theory compactifications on U-folds.

There is a $S L(2, \mathbb{Z})^{7}$ invariant (up to Kähler transformations) superpotential induced by the flux spinor $\mathbb{G}$, which is given by [5]

$$
W=\left.\mathbb{G} \otimes e^{i \mathbb{T}}\right|_{(+;+,+,+;+,+,+)}
$$

with $(+;+,+,+;+,+,+)$ the spinor component selected by the four dimensional $\mathcal{N}=1$ gravitino, and

$$
e^{i \mathbb{T}} \equiv 1+i \mathbb{T}-\mathbb{T} \otimes \mathbb{T}+\ldots
$$

The expression of the superpotential in terms of flux parameters can be found in the Appendix.

In terms of $S L(2, \mathbb{Z})^{7}$ Weyl spinors, the 24 components of the Q-flux allowed by the orientifold involution read

$$
Q_{p}^{a b} \equiv(+; \underline{-,+,+} ; \underline{ \pm, \pm, \pm})
$$

where underlining indicates all possible permutations.

Since we know how $S L(2, \mathbb{Z})^{7}$ transformations act on the fluxes, a systematic use of such actions on eq.(2.10) should lead to the complete algebra involving the $2^{7}$ dual fluxes.

With this purpose in mind, we assign spinor indices to the $X^{a}$ generators. By matching the left and right sides of eq.(2.10) we get 4

$$
X^{i}=(+; \overbrace{+, 0,0}^{i} ; \overbrace{+, 0,0}^{i+3}), \quad X^{i+3}=(+; \overbrace{+, 0,0}^{i} ; \overbrace{-, 0,0}^{i+3}),
$$

where a "zero" in a given position reflects that the generator behaves as a scalar under the action of that particular $S L(2, \mathbb{Z})$.

\footnotetext{
${ }^{4}$ This is the index structure that would be assigned to $X^{a} \equiv\left(Q^{a}\right)_{c}^{b}$ by identifying the structure constants as matrix elements of generators in the adjoint representation. For instance, $X^{1}=\left(Q^{1}\right)_{c}^{b}$ corresponds to a $6 \times 6$ matrix with eight non-vanishing elements given by fixing a plus sign in the $0^{\text {th }}$, $1^{\text {st }}$ and $4^{\text {th }}$ components of the weight vector and keeping the other components unfixed (see table 4 in the Appendix).
} 
Now, it is simple to see that the algebra $(2.10)$ is incomplete. Consider for instance the following commutator in eq.(2.10),

$$
\begin{aligned}
{\left[X^{1}, X^{2}\right]=Q_{3}^{12} X^{3}+Q_{6}^{12} X^{6} } & = \\
& =-(+;+,+,-;+,+,-) X^{3}+(+;+,+,-;+,+,+) X^{6} .
\end{aligned}
$$

The 1.h.s. in this equation is invariant under $S L(2, \mathbb{Z})_{k}$, for $k=3,6$, whereas the r.h.s. is not. Hence, if we insist in consistency under those generators we must modify the right member of the equality.

Acting with $\mathcal{S}_{3,2}$ on the fluxes

$$
\mathcal{S}_{3,2} Q_{3}^{12}=-\tilde{F}^{126}, \quad \mathcal{S}_{3,2} Q_{6}^{12}=\tilde{F}^{123}
$$

with $\tilde{F}_{3}$ defined in eq.(2.8), and introducing a new set of generators, $Z_{i}$

$$
Z_{i}=-(+; \overbrace{-, 0,0}^{i} ; \overbrace{-, 0,0}^{i+3}), \quad Z_{i+3}=(+; \overbrace{-, 0,0}^{i} ; \overbrace{+, 0,0}^{i+3})
$$

the modified invariant commutator is given by,

$$
\left[X^{1}, X^{2}\right]=Q_{3}^{12} X^{3}+Q_{6}^{12} X^{6}-\tilde{F}^{123} Z_{3}-\tilde{F}^{126} Z_{6}
$$

The application of $\mathcal{S}_{6,2}$ would lead to the same conclusion. Actually, we could have envisaged this expression by recalling that a $S L(2, \mathbb{Z})$ scalar built up from two spinors $W_{ \pm}$and $Q_{ \pm}$takes the expression,

$$
W_{+} Q_{-}-W_{-} Q_{+} \equiv W_{p} Q^{p}
$$

While the sixth index in eq. (3.8) is contracted in an invariant way, the third index is not. For the generic case we therefore have the algebra,

$$
\left[X^{a}, X^{b}\right]=-\tilde{F}^{a b p} Z_{p}+Q_{p}^{a b} X^{p}
$$

The same kind of reasoning allows to compute the other commutators, $\left[Z_{a}, X^{b}\right]$ and $\left[Z_{a}, Z_{b}\right]$. For example, applying $\mathcal{S}_{b, 2}$ to the commutator $\left[X^{a}, X^{b}\right]$ and using the rules 
derived above, we obtain $\left[X^{a}, Z_{b+3}\right]$. Thus, by proceeding in the same manner with the other generators, we find a full algebra involving non-geometric and RR 3-form fluxes, 5

$$
\begin{aligned}
{\left[X^{a}, X^{b}\right] } & =-\tilde{F}^{a b p} Z_{p}+Q_{p}^{a b} X^{p} \\
{\left[X^{a}, Z_{b}\right] } & =-Q_{b}^{a p} Z_{p} \\
{\left[Z_{a}, Z_{b}\right] } & =0 .
\end{aligned}
$$

The Jacobi identity of this algebra is given by eq.(2.11) together with the additional constraint,

$$
Q_{p}^{[a b} \tilde{F}^{c] l p}+\tilde{F}^{p[a b} Q_{p}^{c] l}=0 \Leftrightarrow Q F_{3}=0
$$

which can be identified with the D7-brane flux-induced charge cancellation condition, eq.(2.35), with $\sum_{I}\left(p_{k}^{I}\right)^{2}=0$. The fact that the tadpole cancellation conditions arising from this algebra do not contain contributions from localized sources, is consistent with the missing additional generators associated to extra massless vector multiplets. The latter are required to account for the gauge symmetries of the field theories confined to the worldvolume of the branes. In Section 4 we give a detailed discussion on how to extend the algebras of closed string gauge generators, to account also for the open string 6 massless vector fields.

The algebra (3.14) is consistent with other results that have previously appeared in

\footnotetext{
${ }^{5}$ In this work we only consider $F_{3}, H_{3}, Q$ and $P$-fluxes, as discussed in Section 2 Strictly speaking, the above procedure would lead also to the inclusion of additional sets of primed fluxes, $F_{a b c}^{\prime}$ and $\omega_{b c}^{\prime a}$, so that the complete invariant algebra is,

$$
\begin{aligned}
{\left[X^{a}, X^{b}\right] } & =-\tilde{F}^{a b p} Z_{p}+Q_{p}^{a b} X^{p}, \\
{\left[X^{a}, Z_{b}\right] } & =\omega^{\prime b}{ }_{a p} X^{p}-Q_{b}^{a p} Z_{p}, \\
{\left[Z_{a}, Z_{b}\right] } & =-F_{a b p}^{\prime} X^{p}+\omega_{a b}^{\prime p} Z_{p} .
\end{aligned}
$$

${ }^{6}$ With some abuse of language, we generically refer to the "open string sector" to describe the sector which contains the fields on the worldvolume of the branes even if in the generic cases of $(p, q) 7$ branes such fields are not associated to fundamental open strings. We thank A. Uranga for a comment on this point.
} 
the literature. Thus, for example, its type I T-dual version,

$$
\begin{aligned}
{\left[Z_{a}, Z_{b}\right] } & =-\boldsymbol{F}_{a b p} Y^{p}+\omega_{a b}^{p} Z_{p} \\
{\left[Z_{a}, Y^{b}\right] } & =-\omega_{a p}^{b} Y^{p}, \\
{\left[Y^{a}, Y^{b}\right] } & =0
\end{aligned}
$$

where we have made use of Tables 1 and 2 in the Appendix and we have introduced $Z_{a}$ and $Y^{a}$ as the T-duals of $X^{a}$ and $Z_{a}$, respectively, is the algebra found in [33], in the framework of supergravity compactifications on twisted tori. In that context, $Y^{b}$ are the generators associated to the $\mathrm{RR}$ vector field, $C_{\mu}=C_{\mu p} Y^{p}$, obtained by dimensional reduction of the RR 2-form, $C_{2}$. The associated Jacobi identities are (2.17) and the Tdual of (3.15), $\omega \mathbf{F}_{3}=0$, the latter ensuring the cancellation of the D5-brane flux-induced RR charge which arises from the Chern-Simons coupling

$$
\int_{M_{4} \times T^{6}} C_{6} \wedge \omega \boldsymbol{F}_{3}
$$

with

$$
(\omega X)_{p m n_{1} \cdots n_{p-1}}=\omega_{[p m}^{a} X_{\left.n_{1} \cdots n_{p-1}\right] a} .
$$

\subsection{Inclusion of $H_{3}$ and $P$-fluxes}

In deriving the algebra (3.14) we have applied all the generators of $S L(2, \mathbb{Z})^{7}$, except for the ones of S-duality. The latter present some subtleties which we would like to address more carefully in this section. The aim is to extend (3.14) to account also for $H_{3}$ and $P$-fluxes. These appear as $S$-duals of $F_{3}$ and $Q$-fluxes, respectively. Namely,

$$
\mathcal{S}_{0,2} F_{a b c}=H_{a b c}, \quad \mathcal{S}_{0,2} Q_{p}^{a b}=P_{p}^{a b}
$$

Since S-duality manifests in four dimensions as electric-magnetic duality, we find convenient to introduce new magnetic generators, $\bar{Z}_{p}$ and $\bar{X}^{p}$,

$$
\mathcal{S}_{0,2} Z_{p}=\bar{Z}_{p}, \quad \mathcal{S}_{0,2} X^{p}=\bar{X}^{p}
$$

In this "democratic" formulation of the four dimensional gauge algebra, we expect to be able to account also for the S-duality. Indeed, now it is straightforward to obtain the 
S-dual of (3.14),

$$
\begin{aligned}
{\left[\bar{X}^{a}, \bar{X}^{b}\right] } & =P_{p}^{a b} \bar{X}^{p}-\tilde{H}^{a b p} \bar{Z}_{p} \\
{\left[\bar{X}^{a}, \bar{Z}_{b}\right] } & =-P_{b}^{a p} \bar{Z}_{p} \\
{\left[\bar{Z}_{a}, \bar{Z}_{b}\right] } & =0
\end{aligned}
$$

where $\tilde{H}_{3}$ is defined in a similar way than $\tilde{F}_{3}$ in eq.(2.8). The resulting Jacobi identities are the S-dual expressions of (3.15) and (2.11) (see below).

The computation of commutators involving $X^{a}$ or $Z_{a}$ with their magnetic counterparts, $\bar{X}^{a}$ and $\bar{Z}_{a}$, is more subtle. Let us start assuming, for the sake of simplicity, that $F_{3}=$ $H_{3}=0$. By matching spinor indices we require the following dependence on the $\mathrm{Q}$ and P-fluxes,

$$
\left[X^{a}, \bar{X}^{b}\right]=A Q_{p}^{a b} \bar{X}^{p}+B P_{p}^{a b} X^{p}
$$

Antisymmetry of the commutator and eq.(2.11), then gives $A=B=1$. However, if we stick to generators in the adjoint representation,

$$
\left(X^{a}\right)_{p}^{b}=Q_{p}^{a b}, \quad\left(\bar{X}^{a}\right)_{p}^{b}=P_{p}^{a b}
$$

the first term in r.h.s. of $(3.22)$ is not independent of the second one and the commutator can be expressed in the simpler form,

$$
\left[X^{a}, \bar{X}^{b}\right]=Q_{p}^{a b} \bar{X}^{p}
$$

where antisymmetry is ensured by the extra condition,

$$
Q_{p}^{a b} P_{m}^{p c}-P_{p}^{a b} Q_{m}^{p c}=0
$$

Performing $S L(2, \mathbb{Z})_{k}(k=1, \ldots 6)$ transformations as above, $F_{3}$ and $H_{3}$ are reintroduced, so that the fully $S L(2, \mathbb{Z})^{7}$ invariant gauge algebra associated to Kaluza-Klein 
vectors is obtained7. When primed fluxes are set to zero (see footnote 51), it reads

$$
\begin{aligned}
{\left[X^{a}, X^{b}\right] } & =-\tilde{F}^{a b p} Z_{p}+Q_{p}^{a b} X^{p} \\
{\left[X^{a}, Z_{b}\right] } & =-Q_{b}^{a p} Z_{p} \\
{\left[X^{a}, \bar{X}^{b}\right] } & =Q_{p}^{a b} \bar{X}^{p}-\tilde{F}^{a b p} \bar{Z}_{p} \\
{\left[\bar{X}^{a}, Z_{b}\right] } & =\left[X^{a}, \bar{Z}_{b}\right]=-Q_{b}^{a p} \bar{Z}_{p} \\
{\left[\bar{X}^{a}, \bar{X}^{b}\right] } & =P_{p}^{a b} \bar{X}^{p}-\tilde{H}^{a b p} \bar{Z}_{p} \\
{\left[\bar{X}^{a}, \bar{Z}_{b}\right] } & =-P_{b}^{a p} \bar{Z}_{p} \\
{\left[\bar{Z}_{a}, \bar{Z}_{b}\right] } & =\left[Z_{a}, Z_{b}\right]=\left[Z_{a}, \bar{Z}_{b}\right]=0 .
\end{aligned}
$$

As before, the constraints arising from the Jacobi identities of the algebra,

$$
\begin{aligned}
& Q_{p}^{[a b} Q_{l}^{c] p}=0 \\
& P_{p}^{[a b} P_{l}^{c] p}=0 \text {, } \\
& Q_{p}^{[a b} P_{l}^{c] p}=P_{p}^{[a b} Q_{l}^{c] p}=0, \\
& Q_{p}^{[a b} \tilde{F}^{c] l p}+\tilde{F}^{p[a b} Q_{p}^{c] l}=0 \Leftrightarrow Q F_{3}=0, \\
& \tilde{H}^{p[a b} P_{p}^{c] l}+P_{p}^{[a b} \tilde{H}^{c] l p}=0 \Leftrightarrow P H_{3}=0 \text {, } \\
& \begin{array}{c}
Q_{p}^{l[a} \tilde{H}^{b c] p}-P_{p}^{[a b} \tilde{F}^{c] l p}= \\
P_{p}^{l[a} \tilde{F}^{b c] p}-Q_{p}^{[a b} \tilde{H}^{c] l p}=0
\end{array} \Leftrightarrow\left\{\begin{array}{l}
P F_{3}+Q H_{3}=0 \\
Q_{p}^{l[a} \tilde{H}^{b c] p}+Q_{p}^{[a b} \tilde{H}^{c] l p}-P_{p}^{l[a} \tilde{F}^{b c] p}-P_{p}^{[a b} \tilde{F}^{c] l p}=0
\end{array}\right.
\end{aligned}
$$

must be supplemented with eq.(3.25) and,

$$
-Q_{p}^{a b} \tilde{H}^{c l p}+P_{p}^{a b} \tilde{F}^{c l p}+\tilde{H}^{p a b} Q_{p}^{c l}-\tilde{F}^{p a b} P_{p}^{c l}=0
$$

ensuring the antisymmetry of mixed generators. We have recovered in this way, both, the $(p, q)$ 7-brane charge tadpole cancellation conditions, given in eqs.(2.34)-(2.36), and the Bianchi identities, eqs.(3.27)-(3.29), which Q and P-fluxes must satisfy for consistency.

\footnotetext{
${ }^{7}$ Notice also the relations between electric and magnetic generators, $Q_{b}^{a p} \bar{Z}_{p}-P_{b}^{a p} Z_{p}=0$ and $Q_{p}^{a b} \bar{X}^{p}-$ $\tilde{F}^{a b p} \bar{Z}_{p}-P_{p}^{a b} X^{p}+\tilde{H}^{a b p} Z_{p}=0$, ensuring the invariance of the algebra under S-duality transformations. These relations can be proven by making use of the antisymmetry conditions and the fact that the generators transform in the adjoint representation of the algebra [48].
} 
Equations (3.27), (3.28) and a constraint analogous to the last condition in (3.32), were already conjectured in [5] by symmetry arguments. In addition, a constraint $Q_{p}^{[a b} P_{l}^{c] p}+$ $P_{p}^{[a b} Q_{l}^{c] p}=0$ was also conjectured. This has been recently interpreted as a cohomology condition in the deformations of (2.10) by its second cohomology class [47]. Here, however, we observe a stronger requirement, given by eq.(3.29). Not every consistent deformation of the algebra (2.10) appears as a valid deformation. The ten dimensional origin of this constraint, having a RR structure, is unknown to us. However, it is worth noticing that (using identifications to be discussed below) couplings proportional to $Q_{p}^{a b} P_{m}^{p c}$ are found in topological terms of $\mathcal{N}=4$ supergravity actions (see for instance [48]) coupled to gauge vector fields, $\epsilon^{\mu \nu \rho \lambda} A_{\mu a}^{+} A_{\nu b}^{-} A_{\rho c}^{+} A_{\lambda^{m}}^{-}$.

\section{Brane gaugings and Freed-Witten anomalies}

The algebra derived in the previous section is incomplete in several aspects which concern the twisted states of the theory. First, it does not include gauge symmetries arising at loci where the elliptic fiber degenerates. That is, the gauge degrees of freedom in the worldvolume of the 7-branes. Second, it does not contain information on sectors of the theory which are not aligned with 7-brane charges, such as the D3-brane tadpole cancellation requirements.

In this section we address the first of these points, whereas the second one will be partially addressed in the next section.

To illustrate how chiral configurations of branes can be described in the gauging formalism, we find convenient to start considering dual type I compactifications on twisted tori, as described at the end of section 2.1, with magnetized D9-branes [49]. The magnetization simply amounts to introducing some worldvolume fluxes, which can be described as gaugings. Namely, due to the Chern-Simons couplings on the worldvolume of the branes, certain RR axionic scalars shift under D-brane $U(1)$ gauge symmetries (due to the presence of the magnetic fields). This is in fact a gauging.

\footnotetext{
${ }^{8}$ The last condition in (3.32) that we have derived here is actually slightly weaker than the singlet condition conjectured in $[5]$.
} 
A key point is that configurations of magnetized D9-branes have non-trivial FreedWitten (FW) constraints in the presence of bulk fluxes. Some D9-brane configurations are not allowed in the presence of worldvolume fluxes, showing a sort of mutual consistency between open and closed string backgrounds. In particular, in [25] (see also [50, 51, 52]) it was suggested that FW constraints could be understood as the requirements needed to ensure invariance of the effective superpotential under shifts of the $U(1)$ gauged axionic scalars.

Here we propose to study this interrelation between open and closed string fluxes from the point of view of the complete algebra describing both, bulk and worldvolume gaugings. The idea is that FW anomaly cancellation requirements arise as a Jacobi identity for the algebra involving both, the closed string generators and the open string ones associated to $U(1)$ gauge fields on the D9-brane worldvolume.

The final aim will be to extend the algebra (3.26) to account for the gauge generators associated to $(p, q)$ 7-branes. As it will be clear below, once these are included, the tadpole cancellation conditions, involving localized 7-brane sources with non-zero total charge, and the Freed-Witten anomaly cancellation conditions, arise from the Jacobi identities of the algebra.

\subsection{A simple example}

Let us consider a configuration of D6-branes in the presence of NSNS $\mathrm{H}_{3}$ flux. The D6-branes wrap 3-cycles of the internal factorized 6-torus, with wrapping numbers

$$
\left(n_{1}, m_{4}\right) \times\left(n_{2}, m_{5}\right) \times\left(n_{3}, m_{6}\right)
$$

For the sake of simplicity, we turn on only some particular component of $H_{3}$, say $\bar{H}_{423}$. The FW constraint in this case simply leads to impose [25]

$$
m_{4} n_{2} n_{3} \bar{H}_{423}=0 \quad i=1,2,3
$$

Under three T-dualities along the directions $x^{4}, x^{5}$ and $x^{6}, \bar{H}_{423}$ maps to a metric flux, $-\omega_{23}^{4}$ (see Table 2), specifying that the $S^{1}$ parameterized by $x^{4}$ is non-trivially fibered over the 2-torus $T_{\left[x^{2} x^{3}\right]}^{2}$, with first Chern class given by the integer $\bar{H}_{423}$. Hence, 
moving around the rectangle spanned by $x^{2}$ and $x^{3}$ implies picking up a shift in $x^{4}$. This intuition is formalized in the algebra relation between the generators of shifts $Z_{i}$

$$
\left[Z_{2}, Z_{3}\right]=\omega_{23}^{4} Z_{4}
$$

The Freed-Witten constraint (4.1) has a clear interpretation in this picture (see [53]). The quantity $n_{2} n_{3} m_{4}$ counts the charge of D5-branes wrapping $T_{\left[x^{2} x^{3}\right]}^{2}$. But the space parameterized by $x^{2}$ and $x^{3}$ is no longer a valid 2-cycle: because of the above mentioned shift, it has a $S^{1}$ boundary parameterized by $x^{4}$.

These nice geometric interpretations, however, are not always available, so we would like to move on towards an algebraic characterization of such constraints, based on consistency conditions of the gauge algebras.

Let us be a bit specific in our example. By defining $B_{2}=\int_{T_{\left[x^{2} x^{3} x^{5} x^{6}\right]}} C_{6}$ and its dual, $a=\int_{T_{\left[x^{1} x^{4}\right]}^{2}} C_{2}$, we can write the D9 Chern-Simons coupling

$$
\int_{D 9} C_{6} \wedge F \wedge F=m_{4} n_{2} n_{3} \int_{4 d} B_{2} \wedge F
$$

or,

$$
m_{4} n_{2} n_{3} \int_{4 d} A^{\mu} \partial_{\mu} a
$$

where $n_{2}\left(n_{3}\right)$ is the number of times the cycle spanned by $x^{2}\left(x^{3}\right)$ is wrapped by the brane, and $\int_{T_{\left[x^{1} x^{4}\right]}^{2}} F=\frac{m_{4}}{n_{1}}$ is the magnetic flux in the first 2-torus [49]. Thus, as mentioned, the isometry corresponding to a shift in $a$ is gauged by the $U(1)$ gauge transformation of the D9-brane. It is therefore natural to introduce a generator, $X$, in the four dimensional theory associated to these $U(1)$ gauge transformations, and to write down the commutators of $X$ with the generators arising from the closed string modes.

The $U(1)$ gauge bundle is non-trivially fibered over the first torus, with first Chern class given by the integer $m_{4} n_{2} n_{3}$. In analogy with the geometric flux above, there is a gauge transformation when going around the rectangle in $x^{1}$ and $x^{4}$. That is, we should have

$$
\left[Z_{1}, Z_{4}\right]=m_{4} n_{2} n_{3} X
$$


with all other commutators vanishing.

It is easy to see, then, that the corresponding FW constraint

$$
\omega_{23}^{4} m_{4} n_{2} n_{3}=0
$$

results from the Jacobi identities of the algebra.

\subsection{Freed-Witten conditions and gaugings for magnetized D9- branes}

Let us now generalize the type I example of the previous section. For that, we consider a stack of D9 branes, wrapping $n_{i}$ times the 2 -cycle $\left[\omega_{i}\right], i=1,2,3$, and magnetic flux given by

$$
n_{i} \mathcal{F}_{i, i+3} \equiv n_{i} \int_{\left[\omega_{i}\right]} F=m_{i+3} .
$$

In type IIB with O9-planes and D9 magnetized branes, there are Chern-Simons couplings of the gauge field strength to the RR forms,

$$
\int_{D 9} C_{6} \wedge F \wedge F=c_{i} \int_{4 d} B_{2}^{i} \wedge F, \quad \int_{D 9} C_{2} \wedge F \wedge F \wedge F \wedge F=c_{0} \int_{4 d} C_{2} \wedge F,
$$

where $c_{i}$ is the corresponding first Chern class,

$$
\begin{aligned}
& c_{0}=-n \mathcal{F}_{14} \mathcal{F}_{25} \mathcal{F}_{36}=-m_{4} m_{5} m_{6} \\
& c_{1}=n \mathcal{F}_{14}=m_{4} n_{2} n_{3} \\
& c_{2}=n \mathcal{F}_{25}=m_{5} n_{1} n_{3} \\
& c_{3}=n \mathcal{F}_{36}=m_{6} n_{1} n_{2} .
\end{aligned}
$$

In these expressions, $n=n_{1} n_{2} n_{3}$, and we have defined the four dimensional 2-forms

$$
B_{2}^{i}=\int_{\left[\tilde{\omega}_{i}\right]} C_{6} .
$$

These are indeed all the couplings of $F$ to 2-forms allowed by the orientifold projection. Introducing also the four dimensional Hodge duals,

$$
d a^{i}=*_{4} d B_{2}^{i}, \quad d a^{0}=-*_{4} d C_{2}
$$


where the fields $a^{I}$, with $I=0 \ldots 3$, correspond respectively to the RR axions, $\operatorname{Im} S, \operatorname{Im}$ $T_{i}$, the couplings (4.8) can be rewritten as,

$$
c_{i} \int \partial_{\mu} a^{i} A^{\mu}, \quad c_{0} \int \partial_{\mu} a^{0} A^{\mu}
$$

Hence, a shift $a^{i} \rightarrow a^{i}+c_{i} \chi$ in the scalar fields is gauged by the gauge transformation of the diagonal $U(1)$ on the D9-branes. Generalizing the previous example and introducing a gauge generator $X$ for this $U(1)$, we write down the algebra,

$$
\left[Z_{a}, Z_{b}\right]=\omega_{a b}^{p} Z_{p}+n \mathcal{F}_{a b} X+\ldots
$$

where the dots refer to other possible bulk fluxes. Actually, we can do it better and consider multiple $U(1)$ subgroups within the D9-brane gauge group, each one with a generator $X^{I}$. Proceeding as in section 3.1, we obtain the following gauge algebra,

$$
\begin{aligned}
{\left[Z_{a}, Z_{b}\right] } & =\omega_{a b}^{p} Z_{p}+n \mathcal{F}_{a b}^{I} X^{I}-\boldsymbol{F}_{a b p} Y^{p} \\
{\left[Z_{a}, Y^{p}\right] } & =-\omega_{a q}^{p} Y^{q} \\
{\left[Z_{a}, X^{I}\right] } & =\mathcal{F}_{a q}^{I} Y^{q}
\end{aligned}
$$

with Jacobi identities

$$
\begin{aligned}
\omega_{[a b}^{p} \omega_{c] p}^{l} & =0 \\
\omega_{[a b}^{p} n \mathcal{F}_{c] p}^{I} & =0 \\
\boldsymbol{F}_{p[a b} \omega_{c] l}^{p}+\omega_{[a b}^{p} \boldsymbol{F}_{c] l p} & =-n \sum_{I} \mathcal{F}_{[a b}^{I} \mathcal{F}_{c] l}^{I} .
\end{aligned}
$$

This algebra could has been obtained, alternatively, by type I/heterotic duality, from the heterotic algebra found in [54], after adjusting some normalization factors, and identifying the worldvolume magnetic fluxes with abelian Yang-Mills fluxes in the heterotic side.

Let us comment on the new equations, (4.19) and (4.20). The first one, is the standard Freed-Witten anomaly cancellation requirement for magnetized D9-branes. Indeed, it is possible to check that it ensures the invariance of the effective superpotential

$$
W_{O 9}=\int_{\mathrm{T}^{6}} \Omega \wedge\left(F_{3}+\omega J_{c}\right)
$$


under the shifts

$$
J_{c} \rightarrow J_{c}+n \mathcal{F} \chi,
$$

with $J_{c}=C_{2}+i J$.

Equation (4.20), on the other hand, was shown in section 3.1 to correspond to the tadpole cancellation requirement for the D5-brane charge. Now, however, the condition appears sourced by a new term, corresponding to the solitonic charge of D5-brane induced in the worldvolume of the magnetized D9-branes

$$
\int_{M_{4} \times \mathrm{T}^{6}} C_{6} \wedge \omega \boldsymbol{F}_{3}-\int_{D 9} C_{6} \wedge F \wedge F .
$$

In this way, as advanced, the inclusion of new generators in the closed string algebra, corresponding to diagonal $U(1)$ gauge symmetries coming from the open string sector, leads to the correct Freed-Witten anomaly and tadpole cancellation conditions.

In what follows, we apply this procedure to extend the algebra (3.26) in order to account also for possible $(p, q)$ 7-branes.

\subsection{Gaugings and Freed-Witten conditions for F-theory 7-branes}

Let us come back to the main setup considered in this work, i.e. globally non-geometric compactifications of F-theory, and consider the presence of magnetized $(p, q)$ 7-branes, containing an ADE gauge theory in their worldvolume. As we have seen, there are generators associated to the $U(1)$ subgroups of these gauge theories which play an important role in the algebra. We will try to implement these by making use of $\mathrm{T}$ and S-dualities.

Type IIB magnetized $\mathrm{D} 7 k_{k}$-branes can be seen as the T-dual of magnetized D9-branes, with magnetization numbers,

$$
\begin{array}{ll}
D 7_{1}: & (1,0) \times\left(n_{2}, m_{5}\right) \times\left(n_{3}, m_{6}\right), \\
D 7_{2}: & \left(n_{1}, m_{4}\right) \times(1,0) \times\left(n_{3}, m_{6}\right), \\
D 7_{3}: & \left(n_{1}, m_{4}\right) \times\left(n_{2}, m_{5}\right) \times(1,0) .
\end{array}
$$

where six T-dualities are applied along the internal 6-torus. The integers $n_{i}$ and $m_{i}$ now correspond to the magnetization of the $D 7_{k}$-branes, defined accordingly to,

$$
m_{i+3} \int_{\left[\omega_{i}\right]} F=n_{i},
$$


with $\left[\omega_{i}\right] \subset\left[\tilde{\omega}_{k}\right]$.

From the algebra (4.17) it is then simple to obtain the dual type IIB algebra,

$$
\begin{aligned}
{\left[X^{a}, X^{b}\right] } & =-\tilde{F}^{a b p} Z_{p}+Q_{p}^{a b} X^{p}+\left(c_{I}^{k}\right)^{a b} X_{k}^{I}, \\
{\left[X^{a}, Z_{b}\right] } & =-Q_{b}^{a p} Z_{p}, \\
{\left[Z_{a}, Z_{b}\right] } & =\left[Z_{a}, X_{k}^{I}\right]=\left[X_{k}^{I}, X_{j}^{J}\right]=0, \\
{\left[X^{a}, X_{k}^{I}\right] } & =\left(\mathcal{F}_{k}^{I}\right)^{a q} Z_{q},
\end{aligned}
$$

corresponding to the extension of the algebra (3.14). The index $k=1,2,3$ labels the three 2 -torus transverse to the $\mathrm{D} 7 k_{k}$-branes, whereas $I$ runs over the different stacks of branes. The first Chern class associated to each of the stacks is now encoded in the quantities

$$
c_{I}^{1}=n_{2}^{I} n_{3}^{I} \mathcal{F}_{1}^{I}, \quad c_{I}^{2}=n_{1}^{I} n_{3}^{I} \mathcal{F}_{2}^{I}, \quad c_{I}^{3}=n_{1}^{I} n_{2}^{I} \mathcal{F}_{3}^{I},
$$

where

$$
\left(\mathcal{F}_{k}^{I}\right)_{a, a+3}=-\left(\mathcal{F}_{k}^{I}\right)_{a+3, a}=\frac{m_{a+3}^{I}}{n_{a}^{I}} \quad \text { for } k \neq a,
$$

and zero otherwise.

We would like to extend this algebra to account also for $H_{3}, P$-fluxes and general $(p, q)$ 7-branes. For that, we follow the same procedure than in section 3.2. and introduce magnetic dual $U(1)$ generators, $\bar{X}^{I}$, associated to the gauge fields in the worldvolume of the 7-branes. The existence of two worldvolume vectors is consistent with the fact that both, F and D-strings, can end on the 7-brane [46]. The diagonal $U(1)$ gauge symmetry in the worldvolume of a stack of $(p, q) 7_{k}$-branes is generated by the linear combination

$$
p_{k}^{I} X_{k}^{I}+q_{k}^{I} \bar{X}_{k}^{I}
$$

Starting with the algebra (4.25) and acting with the S-duality generators, as in section 3.2, we obtain the extension of the full algebra (3.26), which accounts also for the gauge 
symmetries associated with the $(p, q) 7_{k}$-branes present in the compactification,

$$
\begin{aligned}
{\left[X^{a}, X^{b}\right] } & =-\tilde{F}^{a b p} Z_{p}+Q_{p}^{a b} X^{p}+\sqrt{2} p_{k}^{I}\left(c_{I}^{k}\right)^{a b} X_{k}^{I} \\
{\left[X^{a}, Z_{b}\right] } & =-Q_{b}^{a p} Z_{p} \\
{\left[X^{a}, X_{k}^{I}\right] } & =\sqrt{2} p_{k}^{I}\left(\mathcal{F}_{k}^{I}\right)^{a q} Z_{q} \\
{\left[X^{a}, \bar{X}^{b}\right] } & =Q_{p}^{a b} \bar{X}^{p}-\tilde{F}^{a b p} \bar{Z}_{p}+\sqrt{2} p_{k}^{I}\left(c_{I}^{k}\right)^{a b} \bar{X}_{k}^{I} \\
{\left[\bar{X}^{a}, Z_{b}\right] } & =\left[X^{a}, \bar{Z}_{b}\right]=-Q_{b}^{a p} \bar{Z}_{p} \\
{\left[X^{a}, \bar{X}_{k}^{I}\right] } & =\left[\bar{X}^{a}, X_{k}^{I}\right]=\sqrt{2} p_{k}^{I}\left(\mathcal{F}_{k}^{I}\right)^{a q} \bar{Z}_{q}, \\
{\left[\bar{X}^{a}, \bar{X}^{b}\right] } & =P_{p}^{a b} \bar{X}^{p}-\tilde{H}^{a b p} \bar{Z}_{p}+\sqrt{2} q_{k}^{I}\left(c_{I}^{k}\right)^{a b} \bar{X}_{k}^{I}, \\
{\left[\bar{X}^{a}, \bar{Z}_{b}\right] } & =-P_{b}^{a p} \bar{Z}_{p}, \\
{\left[\bar{X}^{a}, \bar{X}_{k}^{I}\right] } & =\sqrt{2} q_{k}^{I}\left(\mathcal{F}_{k}^{I}\right)^{a q} \bar{Z}_{q},
\end{aligned}
$$

with all the other commutators vanishing. In addition, the following conditions are also required to ensure antisymmetry of the commutators,

$$
\begin{aligned}
& Q_{p}^{a b} P_{m}^{p c}-P_{p}^{a b} Q_{m}^{p c}=0 \\
& -Q_{p}^{a b} \tilde{H}^{c l p}+P_{p}^{a b} \tilde{F}^{c l p}+\tilde{H}^{p a b} Q_{p}^{c l}-\tilde{F}^{p a b} P_{p}^{c l}=0 \\
& \left(\mathcal{F}_{k}^{I}\right)^{a p}\left(q_{k}^{I} Q_{p}^{c d}-p_{k}^{I} P_{p}^{c d}\right)=0 .
\end{aligned}
$$

The Jacobi identities of the algebra can be then summarized in the following sets of constraints,

- Bianchi identities:

$$
\begin{aligned}
& Q_{p}^{[a b} Q_{l}^{c] p}=0, \\
& P_{p}^{[a b} P_{l}^{c] p}=0, \\
& Q_{p}^{[a b} P_{l}^{c] p}=P_{p}^{[a b} Q_{l}^{c] p}=0, \\
& Q_{p}^{l[a} \tilde{H}^{b c] p}+Q_{p}^{[a b} \tilde{H}^{c] l p}-P_{p}^{l[a} \tilde{F}^{b c] p}-P_{p}^{[a b} \tilde{F}^{c] l p}=0,
\end{aligned}
$$


- 7-brane tadpoles:

$$
\begin{aligned}
& \left(Q F_{3}\right)_{k}=-\sum_{I}\left(p_{k}^{I}\right)^{2} d_{k}^{I}, \\
& \left(P H_{3}\right)_{k}=-\sum_{I}\left(q_{k}^{I}\right)^{2} d_{k}^{I}, \\
& \left(P F_{3}+Q H_{3}\right)_{k}=-2 \sum_{I} p_{k}^{I} q_{k}^{I} d_{k}^{I},
\end{aligned}
$$

- Freed-Witten anomalies:

$$
\begin{aligned}
& Q_{p}^{[a b}\left(c_{I}^{k}\right)^{c] p}=0, \\
& P_{p}^{[a b}\left(c_{I}^{k}\right)^{c] p}=0,
\end{aligned}
$$

where we have made use of the wrapping numbers of the $I$-th stack of $7_{k}$-branes, $d_{k}^{I}$, defined as

$$
d_{1}^{I}=m_{5}^{I} m_{6}^{I}, \quad d_{2}^{I}=m_{4}^{I} m_{6}^{I}, \quad d_{3}^{I}=m_{4}^{I} m_{5}^{I} .
$$

Notice that, for non-vanishing $H_{3}$ and $P$-fluxes, the superpotential (2.32) also develops a linear dependence in the axion-dilaton. Invariance under the shifts

$$
\operatorname{Im} S \rightarrow \operatorname{Im} S+c_{0} \chi,
$$

would lead to Freed-Witten anomaly cancellation requirements for magnetized D9-branes with $c_{0} \neq 0$. Here, however, we only consider the presence of 3 and 7 -branes (see footnote 2).

We have obtained, thus, the full gauge algebra associated to Kaluza-Klein and 7-brane vectors in the class of F-theory compactifications described in section 2, The Jacobi identities of the algebra encode all the information relative to the Bianchi identities, tadpole cancellation conditions for $(p, q)$ 7-branes and Freed-Witten anomaly cancellation requirements, corresponding to the electric and magnetic parts of the worldvolume fields. Hence, whereas for the class of fluxes we are considering here, the consistency conditions which the background must satisfy are elusive in a ten dimensional description in terms of local supergravity solutions plus stringy monodromies, the four dimensional gauge algebra captures much of the information of the higher dimensional model. The underlying 
philosophy is that every consistent (holomorphic) gauging of the four dimensional effective supergravity is capturing the dynamics of some light modes of the higher dimensional superstring theory 9 , even if the latter is in the strongly coupled and/or $\alpha^{\prime} \gg 1$ regime. Of course, in the latter case, corrections to the effective supergravity description are expected to be important.

\section{Comparison with gauged supergravity actions}

\section{1 $\mathcal{N}=4$ gauged supergravity}

Before the inclusion of fluxes, the effective four dimensional theory resulting from orientifold compactification on a 6 -torus preserves $\mathcal{N}=4$ supersymmetry. Such is the case for example of F-theory compactified on $K 3 \times T^{4}$, discussed in [6].

The covariant formulation of $\mathcal{N}=4$ gauged supergravity in four dimensions has been proposed in [48]. The big amount of local supersymmetry constrains the only possible deformations of the theory to be gaugings induced by minimal couplings of vector fields to "isometry" generators of the background. The structure constants of the gauge algebra are naturally identified with flux parameters of the higher dimensional compactified theory, as depicted in the previous sections.

The set of possible gaugings of $\mathcal{N}=4$ supergravity, with $n$ extra vector multiplets, is encoded in two embedding tensors of $S L(2, \mathbb{Z}) \times S O(6,6+n ; \mathbb{Z})[48]$

$$
f_{\alpha M N P}, \quad \xi_{\alpha M}
$$

with $\alpha= \pm 1 / 2$ and $M=1 \ldots 12+n$. Supersymmetry and anomaly cancellation requires the emergence of a potential for the scalars living in the vector multiplets

$$
\begin{array}{r}
V_{\mathcal{N}=4}=-\frac{1}{16}\left[f_{\alpha M N P} f_{\beta Q R S} M^{\alpha \beta}\left(\frac{1}{3} M^{M Q} M^{N R} M^{P S}+\left(\frac{2}{3} \eta^{M Q}-M^{M Q}\right) \eta^{N R} \eta^{P S}\right)\right. \\
\left.-\frac{4}{9} f_{\alpha M N P} f_{\beta Q R S} \epsilon^{\alpha \beta} M^{M N P Q R S}+3 \xi_{\alpha}^{M} \xi_{\beta}^{N} M^{\alpha \beta} M_{M N}\right]
\end{array}
$$

\footnotetext{
${ }^{9}$ As pointed out in [55], it may well happen, however, that the higher dimensional solution does not correspond to a compact manifold.
} 
together with a topological term for the vector fields [48]. The indices are lowered and raised with the off-block diagonal metric,

$$
\eta_{M N}=\eta^{M N}=\left(\begin{array}{cc}
0 & \mathbb{I}_{6} \\
\mathbb{I}_{6} & 0
\end{array}\right)
$$

and the antisymmetric tensor $\epsilon^{\alpha \beta}=\epsilon_{\alpha \beta}= \pm 1$. The matrices $M_{M N}$ and $M_{M N P Q R S}$ are given in terms of a $S O(6,6)$ vielbein matrix $\mathcal{V}^{P}{ }_{Q}$ as

$$
M_{M N}=\mathcal{V}^{P}{ }_{M} \mathcal{V}_{N}^{P}, \quad M_{M N P Q R S}=\epsilon_{m n o p q r} \mathcal{V}^{m}{ }_{M} \mathcal{V}_{N}^{n} \mathcal{V}_{P}^{o} \mathcal{V}_{Q}^{p} \mathcal{V}^{q}{ }_{R} \mathcal{V}_{S}^{r}
$$

where the lower-case indices run from 1 to 6.10 In the minima of the scalar potential some of the scalars acquire a mass, reflecting the moduli stabilization induced by the fluxes.

Let us consider first the simplest case consisting on pure super-Poincaré symmetry with no extra vector multiplets $(n=0)$. This is exactly the same situation discussed in section 3, where the $U(1)$ generators of the branes were decoupled from the Kaluza-Klein vectors. In that case, the matrices $M^{M N}$ and $M^{\alpha \beta}$ can be chosen to be parameterized as

$$
M^{M N}=\left(\begin{array}{cc}
g^{m n} & -g^{m k} c_{k n} \\
c_{m k} g^{k n} & g_{m n}-c_{m k} g^{k \ell} c_{\ell n}
\end{array}\right), \quad M^{\alpha \beta}=\frac{1}{\operatorname{Re} S}\left(\begin{array}{cc}
1 & \operatorname{Im} S \\
\operatorname{Im} S & |S|^{2}
\end{array}\right)
$$

where, the tensors $g_{m n}$ and $c_{m n}$ correspond respectively to the metric tensor and the RR 4-form of a toroidal compactification of type IIB supergravity,

$$
d s^{2}=\frac{1}{2} g_{m n} d x^{m} d x^{n}, \quad C_{4}=\frac{1}{2} c_{m n} *_{6}\left(d x^{m} \wedge d x^{n}\right) .
$$

For the particular case of a factorizable torus, these are given in eqs.(2.1) and (2.2). The $\mathbb{Z}_{2} \times \mathbb{Z}_{2}$ symmetry ensuring the factorization of the background breaks the $S O(6,6) \times$ $S L(2, \mathbb{Z})$ global symmetry group to a $[S L(2, \mathbb{Z})]^{7} \subset S O(7,7)$ subgroup, as already described. The action on the embedding tensor is such that $\xi_{\alpha M}$ is projected out completely, whereas only the components

$$
(M \bmod 3) \neq(N \bmod 3) \neq(P \bmod 3)
$$

\footnotetext{
${ }^{10}$ Notice that vielbeins in eq.(5.4) are rotated with respect to those in Ref.48, since we are using a different, off-diagonal, $S O(6,6)$ metric.
} 
of $f_{\alpha M N P}$ survive to the projection. The gauge group generators are then easily identified in terms of the embedding tensor, and the full gauge algebra is given by [48]

$$
\left[Z_{\alpha A}, Z_{\beta B}\right]=\delta_{\beta}^{\rho} f_{\alpha A B}^{P} Z_{\rho P}
$$

leading to general Jacobi identities,

$$
\begin{array}{r}
f_{\alpha[A B}^{P} f_{\beta B] P}^{L}=0 \\
f_{+A B}^{P} f_{-B P}^{L}-f_{-A B}^{P} f_{+B P}^{L}=0
\end{array}
$$

The algebra (3.26), which we have constructed by using modular transformations, exactly matches (5.8), with the extra flux parameters corresponding to primed fluxes, which we have set to zero (see footnote 5). This constitutes a non trivial crosscheck that nongeometric flux compactifications, like the ones described in section 2, lead to consistent gauged supergravities in four dimensions. More precisely, the following dictionary between the flux spinor $\mathbb{G}$ and the projected in components of the embedding tensor can be established

$$
\mathbb{G}_{\left(s_{0}, s_{1}, s_{2}, s_{3}, s_{4}, s_{5}, s_{6}\right)}=\operatorname{Sign}\left(s_{0} M_{1} M_{2} M_{3}\right) f_{s_{0},\left|M_{1}\right|,\left|M_{2}\right|,\left|M_{3}\right|}
$$

where $s_{i}= \pm 1 / 2$ and

$$
\begin{aligned}
M_{k}=\frac{1}{4}\left[(k+9)\left(2 s_{k}-1\right)\left(2 s_{k+3}-1\right)-(k+6)\left(2 s_{k}-1\right)\left(2 s_{k+3}+1\right)+\right. \\
\left.+(k+3)\left(2 s_{k}+1\right)\left(2 s_{k+3}+1\right)+k\left(2 s_{k}+1\right)\left(2 s_{k+3}-1\right)\right] .
\end{aligned}
$$

Equivalently, in terms of $[S O(2)]^{3}$ covariant tensors,

$$
\begin{aligned}
f_{+a}{ }^{b c} & =-\frac{1}{2} Q_{a}^{b c}, & f_{+}{ }^{a b c} & =\frac{1}{2} \tilde{F}^{a b c} \\
f_{-a}{ }^{b c} & =-\frac{1}{2} P_{a}^{b c}, & f_{-}{ }^{a b c} & =\frac{1}{2} \tilde{H}^{a b c}
\end{aligned}
$$

and similarly for the primed fluxes. Here, $a, b, c=1 \ldots 6$, and indices are raised with the $S O(6,6)$ metric $\eta_{M N}$, given in eq.(5.3). Notice that electric (magnetic) gaugings do not necessarily correspond to NSNS (RR) fluxes, contrary to what it is sometimes stated in the supergravity literature. 
We can now easily extend this dictionary to the case on which $(p, q) 7$-branes are also present. Notice that $\mathcal{N}=4$ supersymmetry allows, at most, for a single class of 7 branes wrapping one of the three 4-cycles of the factorized 6-torus. There are electric and magnetic generators, $X^{I}$ and $\bar{X}^{I}, I=0 \ldots n$, corresponding to diagonal $U(1)$ symmetries in the worldvolume of the the 7-branes. The uppercase indices of the embedding tensor, $f_{\alpha M N P}$, now run from 1 to $12+n$. And, in particular, looking at the structure of (4.29), we see that we can accommodate the 7-brane gaugings in the following components of the embedding tensor,

$$
\frac{1}{2}\left(c_{I}\right)^{a b}=-\frac{1}{p^{I}} f_{+(12+I)}^{a b}=-\frac{1}{q^{I}} f_{-(12+I)}^{a b},
$$

where $\left(c_{I}\right)^{a b}$ is the gauging parameter defined in (4.26) and (4.27), for the relevant 7-brane.

\section{$5.2 \mathcal{N}=1$ structure and D3-brane sector}

We have just observed that the algebra (3.26) fits perfectly within a truncation of a $\mathcal{N}=4$ gauged supergravity algebra. This is because in deriving (3.26) we have actually made use of the symmetries and dualities of $\mathcal{N}=4$ supergravity. We therefore, do not expect the algebra (3.26) to contain intrinsic information of the $\mathcal{N}=1$ supersymmetry. A first hint of this is that the tadpole cancellation condition for the D3-brane charge, given in eq.(2.7), does not arise from the quadratic constraints, eqs.(5.9)-(5.10), of the algebra. Indeed, D3-branes are special in that they couple electrically to the RR 4-form, $C_{4}$, which only fills the non-compact directions. In this sense, the cancellation of the D3-brane charge is not a topological condition which can be derived from the algebra (4.29).

A detailed analysis of the scalar potential (5.2) moreover shows that the truncation (5.7) together with the Jacobi identities of the $\mathcal{N}=4$ algebra guarantee a $\mathcal{N}=1$ structure for $(5.2)$

$$
V_{\mathcal{N}=1}=e^{K}\left(\sum_{\mathbb{T}_{i}}\left(\mathbb{T}_{i}+\overline{\mathbb{T}}_{i}\right)^{2}\left|D_{\mathbb{T}_{i}} W\right|^{2}-3|W|^{2}\right),
$$

with $K$ the Kähler potential of the factorized torus, given in eq.(2.4) and $W$ the superpotential in eq.(2.32).

\footnotetext{
${ }^{11}$ We thank the authors of [59] for noticing an error in previous versions which was leading to an apparent disagreement between both scalar potentials.
} 


\section{Summary and Outlook}

The main aim of the this work was to advance in the understanding of string compactifications when fluxes, geometric and non-geometric, are switched on. Different dualities suggest the presence of a huge collection of background fluxes. For instance, the setup considered in our work (where an initial orientifold compactification on factorized tori is enforced) allows for as many as $2^{7}$ of such fluxes, encoded in $S L(2, \mathbb{Z})^{7}$ spinorial representations. Flux parameters manifest as gaugings in four dimensions. The gauged four dimensional supergravity theory contains information about the fully stringy aspects of the original theory and, generically, it is not just a compactification of ten dimensional supergravity. As a consequence, identifying the ten dimensional origin of the gaugings is far from being straightforward. One goal of our study was to show that half of these fluxes can be associated to global, non-geometric, compactifications of F-theory. The compactification manifold appears to be an U-fold [8, 7] where local patches are glued by performing $\mathrm{T}$ and S-duality transformations. We illustrated such ideas through an explicit example, which, by using $\mathrm{S}$ and $\mathrm{T}$ dualities, can be mapped into a standard Type IIB compactification.

Comprehension of the algebraic gauge structure of the effective theories arising from superstring theory compactifications is of great importance for understanding both, the four dimensional physics and the vacuum structure of the higher dimensional theory. By means of a systematic use of duality transformations, we were able to derive the full $\mathcal{N}=4$ algebra satisfied by gauge generators related to Kaluza-Klein vector fields. Jacobi identities of such algebra provide a consistent way of finding constraints satisfied by fluxes.

Moreover, we have shown that the 7-brane sector (or more generically, the open string sector or twisted sector) can also be settled in a similar framework, where branes are described in the gauging formalism. We found that the Jacobi identities of the algebra, mixing bulk generators and generators of $U(1)$ gauge groups on the branes, lead to FreedWitten like requirements, constraining the allowed brane configurations.

Comparison with $\mathcal{N}=4$ gauged supergravity theories allowed us to establish a dictionary between fluxes and gaugings and to relate Jacobi identities to quadratic constraints 
on gaugings, necessary for consistency of supergravity theories. In particular, some of these constraints appear to be stronger than those previously discussed in the literature. There appear to be also extra requirements that are not captured by the $\mathcal{N}=4$ algebra and which can be associated to D3-brane charges. It would be interesting to see if these extra requirements could be obtained as Jacobi identities of an extended algebra, presumably, associated to $\mathcal{N}=1$ gauged supergravity theory. This fact deserves further investigation.

There are several other directions which this work leaves open. For instance, we have not addressed the exploration of the vacuum structure associated to the algebra (4.29). Some first steps along this direction has been taken in [56, 47], using techniques of computational algebraic geometry. We find it would be interesting to know how the results of those investigations are modified by the stronger constraints we found here, or by including in the algebra the gauge generators corresponding to the open string/twisted sector. In particular, it is not transparent to us if solutions, beyond the family we have described in section 2.3 ( related to standard type IIB compactifications with 3-form fluxes by four T-dualities and a S-duality rotation), do exist.

It has also been also shown in [57] that generalized geometry can provide a local geometrical definition of the NSNS flux parameters appearing in the four dimensional effective gauged supergravity. Here we have presented a procedure to extend these algebras in order to account also for the RR degrees of freedom. Definitively, it would be interesting to understand the new flux parameters appearing in these extended algebras from the point of view of exceptional generalized geometry [58].

We believe that the combination of general flux backgrounds, like the ones considered here, with suitable chiral configurations of F-theory 7-branes, is a good road towards the construction of phenomenological models, providing powerful tools for addressing not only the right spectrum of particles, but also moduli stabilization or supersymmetry breaking. Hopefully, the algebras we have presented in this work will serve to construct and analyze phenomenological F-theory compactifications to four dimensions in the near future. 


\section{Acknowledgments}

We thank E. Andrés, L. Carlevaro, A. Font, A. Guarino and F. Marchesano for useful discussions and comments and, in particular, A. Uranga who participated in the preliminary steps of this work. The work of P.G.C. is supported by the European Union through an Individual Marie-Curie IEF. Additional support comes from the contracts ANR-05-BLAN-0079-02, MRTN-CT-2004-005104, MRTN-CT-2004-503369, MEXT-CT2003-509661 and CNRS PICS \# 2530, 3059, 3747. G.A. work is partially supported by PIP5231 (CONICET)and grant 06/C225 (UNC). A.R. thanks ICTP for partial support. 


\section{A Background fluxes}

Here we collect tables containing the RR and NSNS flux parameters surviving the $\mathbb{Z}_{2} \times \mathbb{Z}_{2}$ and orientifold projections, and their tensorial structure in different T-dual formulations, in the notation of [5].

\begin{tabular}{cccc|cccc} 
IIB $/$ O3 & IIA/O6 & IIB/O9 & flux & IIB/O3 & IIA/O6 & IIB/O9 & flux \\
\hline$F_{123}=-\tilde{F}^{456}$ & $F_{0}$ & $-\boldsymbol{F}_{456}$ & $-m$ & $H_{123}=-\tilde{H}^{456}$ & $R^{123}$ & $\boldsymbol{R}^{123}$ & $\bar{h}_{0}$ \\
$F_{423}=\tilde{F}^{156}$ & $F_{14}$ & $\boldsymbol{F}_{156}$ & $-q_{1}$ & $H_{423}=\tilde{H}^{156}$ & $-Q_{4}^{23}$ & $\boldsymbol{R}^{423}$ & $-\bar{a}_{1}$ \\
$F_{153}=\tilde{F}^{426}$ & $F_{25}$ & $\boldsymbol{F}_{426}$ & $-q_{2}$ & $H_{153}=\tilde{H}^{426}$ & $-Q_{5}^{31}$ & $\boldsymbol{R}^{153}$ & $-\bar{a}_{2}$ \\
$F_{126}=\tilde{F}^{453}$ & $F_{36}$ & $\boldsymbol{F}_{453}$ & $-q_{3}$ & $H_{126}=\tilde{H}^{453}$ & $-Q_{6}^{12}$ & $\boldsymbol{R}^{126}$ & $-\bar{a}_{3}$ \\
$F_{156}=-\tilde{F}^{423}$ & $F_{2536}$ & $-\boldsymbol{F}_{423}$ & $e_{1}$ & $H_{156}=-\tilde{H}^{423}$ & $-\omega_{56}^{1}$ & $\boldsymbol{R}^{156}$ & $-a_{1}$ \\
$F_{426}=-\tilde{F}^{153}$ & $F_{1436}$ & $-\boldsymbol{F}_{153}$ & $e_{2}$ & $H_{426}=-\tilde{H}^{153}$ & $-\omega_{64}^{2}$ & $\boldsymbol{R}^{426}$ & $-a_{2}$ \\
$F_{453}=-\tilde{F}^{126}$ & $F_{1425}$ & $-\boldsymbol{F}_{126}$ & $e_{3}$ & $H_{453}=-\tilde{H}^{126}$ & $-\omega_{45}^{3}$ & $\boldsymbol{R}^{453}$ & $-a_{3}$ \\
$F_{456}=\tilde{F}^{123}$ & $F_{142536}$ & $\boldsymbol{F}_{123}$ & $-e_{0}$ & $H_{456}=\tilde{H}^{123}$ & $\bar{H}_{456}$ & $\boldsymbol{R}^{456}$ & $h_{0}$
\end{tabular}

Table 1: Type IIB/O3 3-form RR (left) and NSNS (right) fluxes and their T-duals.

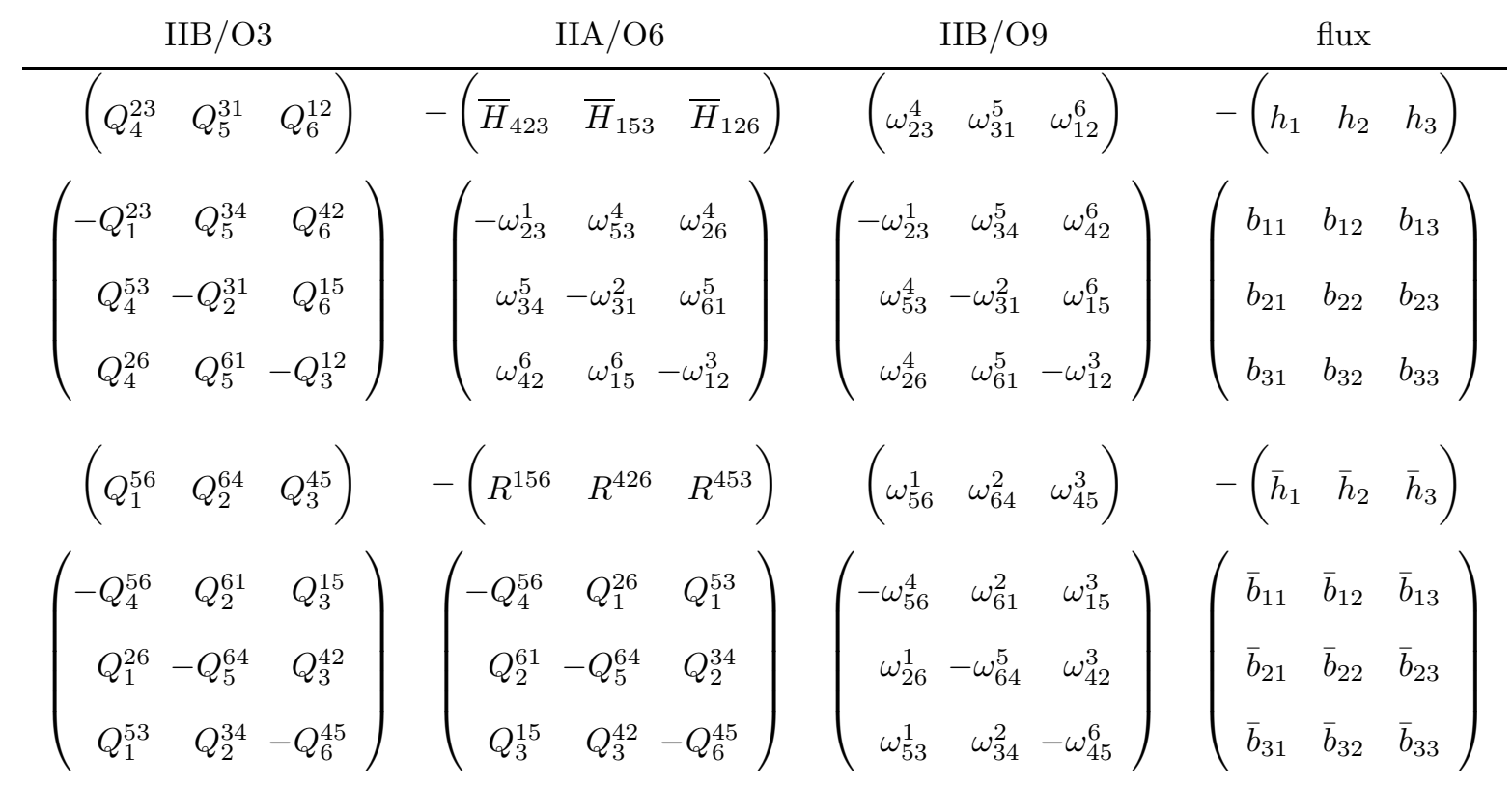

Table 2: Type IIB/O3 NSNS Q-fluxes and their T-duals. 


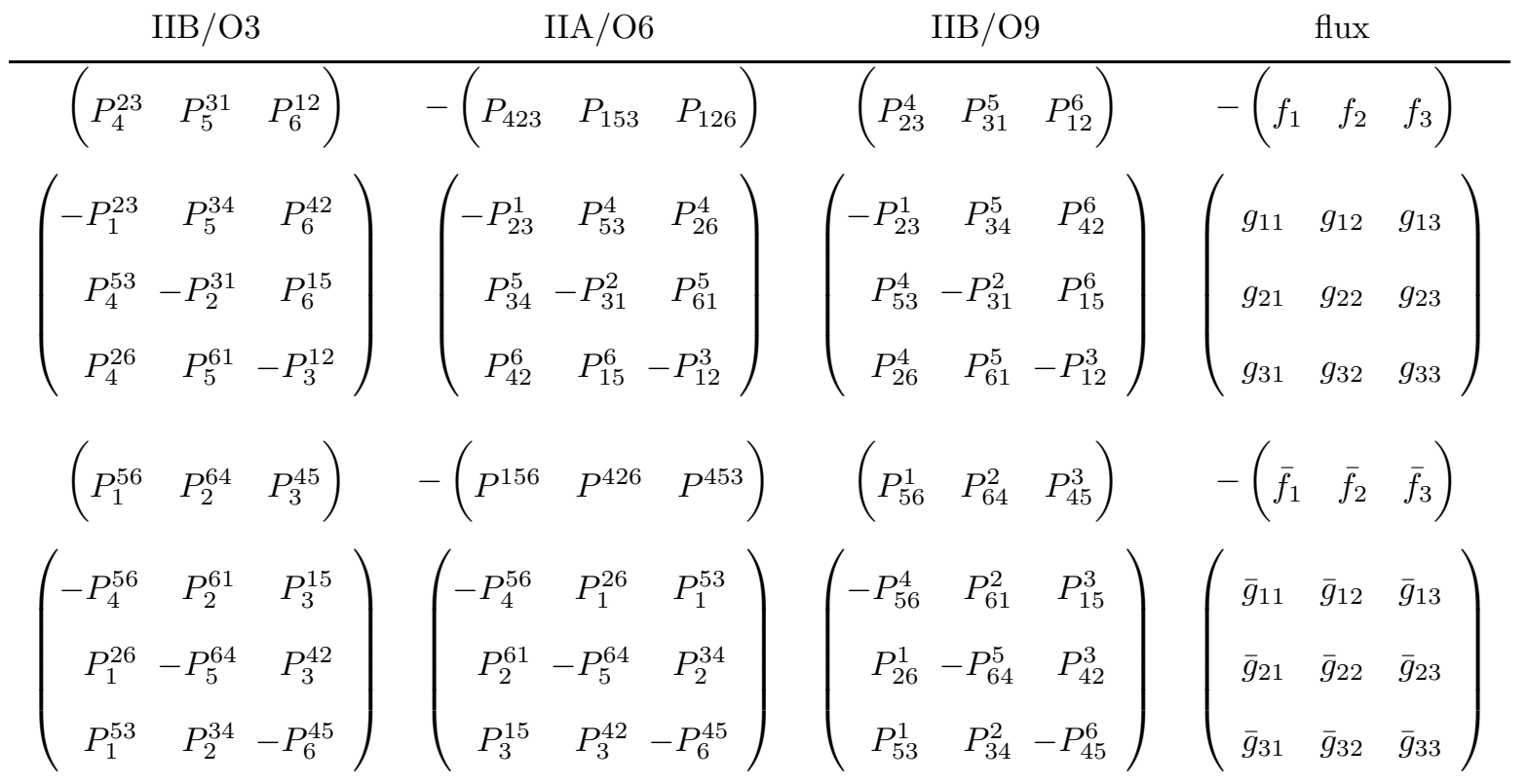

Table 3: Type IIB/O3 RR P-fluxes and their T-duals.

We also state the superpotential (3.4), in terms of the flux parameters defined in table 4 ,

$$
\begin{aligned}
W_{\text {Flux }} & =e_{0}-i \sum_{i=1}^{3} h_{i} T_{i}+\frac{1}{2} \sum_{l \neq m \neq n} h_{l}^{\prime} T_{m} T_{n}+i e_{0}^{\prime} T_{1} T_{2} T_{3} \\
& +\left(i h_{0}-\sum_{i=1}^{3} f_{i} T_{i}-\frac{i}{2} \sum_{l \neq m \neq n} f_{l}^{\prime} T_{m} T_{n}-h_{0}^{\prime} T_{1} T_{2} T_{3}\right) S \\
& +\sum_{i=1}^{3}\left[\left(-a_{i}+i \sum_{j=1}^{3} g_{i j} T_{j}-\frac{1}{2} \sum_{l \neq m \neq n} g_{i l}^{\prime} T_{m} T_{n}+i a_{i}^{\prime} T_{1} T_{2} T_{3}\right) S\right. \\
& \left.+i e_{i}-\sum_{j=1}^{3} b_{i j} T_{j}-\frac{i}{2} \sum_{l \neq m \neq n} b_{i l}^{\prime} T_{m} T_{n}-e_{i}^{\prime} T_{1} T_{2} T_{3}\right] U_{i} \\
& +\frac{1}{2} \sum_{r \neq s \neq t}\left[\left(i \bar{a}_{r}+\sum_{j=1}^{3} \bar{g}_{r j} T_{j}+\frac{i}{2} \sum_{l \neq m \neq n} \bar{g}_{r l}^{\prime} T_{m} T_{n}-\bar{a}_{r}^{\prime} T_{1} T_{2} T_{3}\right) S\right. \\
& \left.-q_{r}+i \sum_{j=1}^{3} \bar{b}_{r j} T_{j}-\frac{1}{2} \sum_{l \neq m \neq n} \bar{b}_{r l}^{\prime} T_{m} T_{n}+i q_{r}^{\prime} T_{1} T_{2} T_{3}\right] U_{s} U_{t} \\
& +\left[-\left(\bar{h}_{0}+i \sum_{j=1}^{3} \bar{f}_{j} T_{j}-\frac{1}{2} \sum_{l \neq m \neq n} \bar{f}_{l}^{\prime} T_{m} T_{n}+i \bar{h}_{0}^{\prime} T_{1} T_{2} T_{3}\right) S\right. \\
& \left.+i m+\sum_{j=1}^{3} \bar{h}_{j} T_{j}+\frac{i}{2} \sum_{l \neq m \neq n} \bar{h}_{l}^{\prime} T_{m} T_{n}-m^{\prime} T_{1} T_{2} T_{3}\right] U_{1} U_{2} U_{3}
\end{aligned}
$$




\begin{tabular}{|c|c|c|c|}
\hline Flux parameter & Weight & Flux parameter & Weight \\
\hline $\bar{h}_{0}^{\prime}$ & $(-,-,-,-,-,-,-)$ & $e_{0}$ & $(+,+,+,+,+,+,+)$ \\
\hline$h_{0}$ & $\underset{i}{(-,+,+,+,+,+,+)}$ & $m^{\prime}$ & $(+,-,-,-,-,-,-)$ \\
\hline$-h_{i}$ & $(+, \overbrace{-,+,+}^{+,+,+,} \underbrace{}_{j}$ & $-\bar{f}_{i}^{\prime}$ & $(-, \overbrace{+,-,-},-,-,-)$ \\
\hline$e_{j}$ & $(+,+,+,+, \overbrace{-,+,+}^{i})$ & $\bar{a}_{j}^{\prime}$ & $(-,-,-,-, \overbrace{i}^{+,-,-})$ \\
\hline $\bar{h}_{i}^{\prime}$ & $(+, \overbrace{+,-,-}^{-},-,-,-)$ & $f_{i}$ & $(-, \overbrace{-,+,+}^{+},+\underbrace{+,+}_{j})$ \\
\hline$q_{j}^{\prime}$ & $-(\overbrace{+,-,-}^{+})$ & $a_{j}$ & $\begin{array}{c}(-,+,+,+, \overbrace{-,+,+} \overbrace{i}^{j} \\
\underbrace{j})\end{array}$ \\
\hline $\bar{g}_{j i}^{\prime}$ & $(-, \overparen{F}$ & $b_{j i}$ & $(+, \overbrace{-,+,+}, \overbrace{-,+,+}^{j})$ \\
\hline$a_{j}^{\prime}$ & $(-,-,-,-, \underbrace{-1}_{i}$ & $q_{j}$ & $\begin{array}{c}(+,+,+,+, \overbrace{+,-,-}^{+}) \\
\underbrace{i} \overbrace{}^{j})\end{array}$ \\
\hline$-g_{j i}$ & $(-, \overbrace{-,+,+}^{-}, \overbrace{-,+,+}^{j})$ & $-\bar{b}_{j i}^{\prime}$ & $(+, \overbrace{+,-,-}^{(+,-,-})$ \\
\hline$-\bar{a}_{j}$ & $\begin{array}{c}(-,+,+,+, \overbrace{+,-,-}^{+\overbrace{}^{j}}) \\
\overbrace{}^{i} \overbrace{}^{j})\end{array}$ & $-e_{j}^{\prime}$ & $\begin{array}{c}(+,-,-,-, \overbrace{-,+,+}^{-}) \\
\overbrace{}^{i} \overbrace{}^{j} \overbrace{}^{i})\end{array}$ \\
\hline$-\bar{b}_{j i}$ & $(+, \overbrace{-,+,+}, \overbrace{+,-,-})$ & $-g_{j i}^{\prime}$ & $(-, \overbrace{+,-,-}, \overbrace{-,+,+})$ \\
\hline$-m$ & $\begin{array}{c}(+,+,+,+,-,-,-) \\
\underbrace{j} \underbrace{j}\end{array}$ & $-h_{0}^{\prime}$ & $\underbrace{(-,-,-,-,+,+,+)}_{i} \underbrace{j}$ \\
\hline$b_{j i}^{\prime}$ & $(+, \overbrace{+,-,-}, \overbrace{-,+,+}^{-})$ & $\bar{g}_{j i}$ & $(-, \overbrace{\substack{-,+,+i}}, \overbrace{+,-,-})$ \\
\hline$f_{i}^{\prime}$ & $(-, \overbrace{+,-,-},+,+,+)$ & $\bar{h}_{i}$ & $(+, \overbrace{-,+,+},-,-,-)$ \\
\hline$-e_{0}^{\prime}$ & $(+,-,-,-,+,+,+)$ & $-\bar{h}_{0}$ & $(-,+,+,+,-,-,-)$ \\
\hline$-\bar{f}_{i}$ & $(-, \overbrace{-,+,+}^{\imath},-,-,-)$ & $-h_{i}^{\prime}$ & $(+, \overbrace{+,-,-}^{\imath},+,+,+)$ \\
\hline
\end{tabular}

Table 4: Spinorial embedding of the background fluxes. The weights in each column correspond to one of the two Weyl spinors on which the set of fluxes $\mathbb{G}$ can be decomposed. 


\section{References}

[1] M. Grana, "Flux compactifications in string theory: A comprehensive review," Phys. Rept. 423 (2006) 91 arXiv:hep-th/0509003; M. R. Douglas and S. Kachru, "Flux compactification," Rev. Mod. Phys. 79 (2007) 733 arXiv:hep-th/0610102]; R. Blumenhagen, B. Kors, D. Lust and S. Stieberger, "Four-dimensional String Compactifications with D-Branes, Orientifolds and Fluxes," Phys. Rept. 445, 1 (2007) arXiv:hep-th/0610327.

[2] S. Gukov, C. Vafa and E. Witten, "CFT's from Calabi-Yau four-folds," Nucl. Phys. B 584 (2000) 69 [Erratum-ibid. B 608 (2001) 477] arXiv:hep-th/9906070].

[3] J. Scherk and J. H. Schwarz, "How To Get Masses From Extra Dimensions," Nucl. Phys. B 153 (1979) 61.

[4] J. Shelton, W. Taylor and B. Wecht, "Nongeometric flux compactifications," JHEP 0510 (2005) 085, hep-th/0508133.

[5] G. Aldazabal, P. G. Camara, A. Font and L. E. Ibanez, "More dual fluxes and moduli fixing," JHEP 0605, 070 (2006) [arXiv:hep-th/0602089].

[6] A. Sen, "F-theory and Orientifolds," Nucl. Phys. B 475 (1996) 562 [arXiv:hep-th/9605150].

[7] A. Kumar and C. Vafa, "U-manifolds," Phys. Lett. B 396, 85 (1997) arXiv:hep-th/9611007.

[8] C. M. Hull and A. Catal-Ozer, "Compactifications with S-duality twists," JHEP 0310 (2003) 034 arXiv:hep-th/0308133; C. M. Hull, "A geometry for non-geometric string backgrounds," JHEP 0510 (2005) 065 arXiv:hep-th/0406102].

[9] R. A. Reid-Edwards and B. Spanjaard, "N=4 Gauged Supergravity from Duality-Twist Compactifications of String Theory," arXiv:0810.4699 [hep-th]; R. A. Reid-Edwards, "Geometric and non-geometric compactifications of IIB supergravity," arXiv:hep-th/0610263.

[10] C. Beasley, J. J. Heckman and C. Vafa, "GUTs and Exceptional Branes in F-theory I," arXiv:0802.3391 [hep-th]; C. Beasley, J. J. Heckman and C. Vafa, "GUTs and Exceptional Branes in F-theory - II: Experimental Predictions," arXiv:0806.0102 [hep-th]; J. J. Heckman and C. Vafa, "F-theory, GUTs, and the Weak Scale," arXiv:0809.1098 [hepth]; J. J. Heckman and C. Vafa, "From F-theory GUTs to the LHC," arXiv:0809.3452 
[hep-ph]; J. J. Heckman and C. Vafa, "Flavor Hierarchy From F-theory," arXiv:0811.2417 [hep-th].

[11] R. Donagi and M. Wijnholt, "Model Building with F-Theory," arXiv:0802.2969 [hep-th]; R. Donagi and M. Wijnholt, "Breaking GUT Groups in F-Theory," arXiv:0808.2223 [hepth]; M. Wijnholt, "F-Theory, GUTs and Chiral Matter," arXiv:0809.3878 [hep-th].

[12] H. Hayashi, R. Tatar, Y. Toda, T. Watari and M. Yamazaki, "New Aspects of HeteroticF Theory Duality," Nucl. Phys. B 806, 224 (2009) arXiv:0805.1057 [hep-th]]; R. Tatar and T. Watari, "GUT Relations from String Theory Compactifications," arXiv:0806.0634 [hep-th].

[13] L. Aparicio, D. G. Cerdeno and L. E. Ibanez, "Modulus-dominated SUSY-breaking soft terms in F-theory and their test at LHC," JHEP 0807, 099 (2008) arXiv:0805.2943 [hep$\mathrm{ph}]$.

[14] J. J. Heckman, J. Marsano, N. Saulina, S. Schafer-Nameki and C. Vafa, "Instantons and SUSY breaking in F-theory," arXiv:0808.1286 [hep-th]; J. Marsano, N. Saulina and S. Schafer-Nameki, "Gauge Mediation in F-Theory GUT Models," arXiv:0808.1571 [hepth]; J. Marsano, N. Saulina and S. Schafer-Nameki, "An Instanton Toolbox for F-Theory Model Building," arXiv:0808.2450 [hep-th].

[15] A. Font and L. E. Ibanez, "Yukawa Structure from U(1) Fluxes in F-theory Grand Unification," arXiv:0811.2157 [hep-th].

[16] J. F. G. Cascales and A. M. Uranga, "Branes on generalized calibrated submanifolds," JHEP 0411 (2004) 083 arXiv:hep-th/0407132.

[17] P. G. Camara, L. E. Ibanez and A. M. Uranga, "Flux-induced SUSY-breaking soft terms on D7-D3 brane systems," Nucl. Phys. B 708 (2005) 268 [hep-th/0408036]; A. Font and L. E. Ibanez, "SUSY-breaking soft terms in a MSSM magnetized D7-brane model," JHEP 0503 (2005) 040 arXiv:hep-th/0412150].

[18] D. Lust, P. Mayr, S. Reffert and S. Stieberger, "F-theory flux, destabilization of orientifolds and soft terms on D7-branes," Nucl. Phys. B 732, 243 (2006) arXiv:hep-th/0501139;

[19] J. Gomis, F. Marchesano and D. Mateos, "An open string landscape," JHEP 0511 (2005) 021 arXiv:hep-th/0506179. 
[20] L. Martucci, "D-branes on general N = 1 backgrounds: Superpotentials and D-terms," JHEP 0606 (2006) 033 arXiv:hep-th/0602129.

[21] A. P. Braun, A. Hebecker, C. Ludeling and R. Valandro, "Fixing D7 Brane Positions by F-Theory Fluxes," arXiv:0811.2416 [hep-th].

[22] K. Becker, M. Becker, C. Vafa and J. Walcher, "Moduli stabilization in non-geometric backgrounds," Nucl. Phys. B 770 (2007) 1 [arXiv:hep-th/0611001].

[23] A. Micu, E. Palti and G. Tasinato, "Towards Minkowski vacua in type II string compactifications," JHEP 0703 (2007) 104 arXiv:hep-th/0701173].

[24] D. S. Freed and E. Witten, "Anomalies in string theory with D-branes," arXiv:hep-th/9907189.

[25] P. G. Camara, A. Font and L. E. Ibanez, "Fluxes, moduli fixing and MSSM-like vacua in a simple IIA orientifold," JHEP 0509 (2005) 013 [arXiv:hep-th/0506066].

[26] S. B. Giddings, S. Kachru and J. Polchinski, "Hierarchies from fluxes in string compactifications," Phys. Rev. D 66, 106006 (2002) [arXiv:hep-th/0105097.

[27] I. R. Klebanov and M. J. Strassler, "Supergravity and a confining gauge theory: Duality cascades and chiSB-resolution of naked singularities," JHEP 0008 (2000) 052 arXiv:hep-th/0007191].

[28] S. Kachru, J. Pearson and H. L. Verlinde, "Brane/flux annihilation and the string dual of a non-supersymmetric field theory," JHEP 0206 (2002) 021 arXiv:hep-th/0112197].

[29] A. Dabholkar and C. Hull, "Duality twists, orbifolds, and fluxes," JHEP 0309 (2003) 054 arXiv:hep-th/0210209].

[30] S. Hellerman, J. McGreevy and B. Williams, "Geometric constructions of nongeometric string theories," JHEP 0401, 024 (2004) arXiv:hep-th/0208174.

[31] A. Flournoy, B. Wecht and B. Williams, "Constructing nongeometric vacua in string theory," Nucl. Phys. B 706, 127 (2005) arXiv:hep-th/0404217.

[32] D. Vegh and J. McGreevy, "Semi-Flatland," JHEP 0810 (2008) 068 [arXiv:0808.1569 [hepth]]. 
[33] G. Dall'Agata and S. Ferrara, "Gauged supergravity algebras from twisted tori compactifications with fluxes," Nucl. Phys. B717 (2005) 223, hep-th/0502066.

[34] F. Marchesano and W. Schulgin, "Non-geometric fluxes as supergravity backgrounds," Phys. Rev. D 76 (2007) 041901 arXiv:0704.3272 [hep-th]].

[35] A. R. Frey and M. Grana, "Type IIB solutions with interpolating supersymmetries," Phys. Rev. D 68 (2003) 106002 arXiv:hep-th/0307142.

[36] G. Dall'Agata, "On supersymmetric solutions of type IIB supergravity with general fluxes," Nucl. Phys. B 695 (2004) 243 arXiv:hep-th/0403220.

[37] C. Vafa and E. Witten, "Dual string pairs with $\mathrm{N}=1$ and $\mathrm{N}=2$ supersymmetry in four dimensions," Nucl. Phys. Proc. Suppl. 46, 225 (1996) arXiv:hep-th/9507050.

[38] P. Meessen and T. Ortin, "An $\mathrm{Sl}(2, \mathrm{Z})$ multiplet of nine-dimensional type II supergravity theories," Nucl. Phys. B 541, 195 (1999) arXiv:hep-th/9806120].

[39] G. Dall'Agata, K. Lechner and M. Tonin, " $\mathrm{D}=10, \mathrm{~N}=$ IIB supergravity: Lorentz-invariant actions and duality," JHEP 9807, 017 (1998) arXiv:hep-th/9806140.

[40] E. Eyras and Y. Lozano, "Exotic branes and nonperturbative seven branes," Nucl. Phys. B 573, 735 (2000) arXiv:hep-th/9908094.

[41] E. A. Bergshoeff, M. de Roo, S. F. Kerstan, T. Ortin and F. Riccioni, "IIB ninebranes," JHEP 0606 (2006) 006 arXiv:hep-th/0601128]; E. A. Bergshoeff, J. Hartong, T. Ortin and D. Roest, "Seven-branes and supersymmetry," JHEP 0702 (2007) 003 arXiv:hep-th/0612072].

[42] K. Dasgupta, G. Rajesh and S. Sethi, "M theory, orientifolds and G-flux," JHEP 9908 (1999) 023 arXiv:hep-th/9908088.

[43] M. Grana and J. Polchinski, "Gauge / gravity duals with holomorphic dilaton," Phys. Rev. D 65 (2002) 126005 arXiv:hep-th/0106014.

[44] D. Baumann, A. Dymarsky, I. R. Klebanov, J. M. Maldacena, L. P. McAllister and A. Murugan, "On D3-brane potentials in compactifications with fluxes and wrapped D-branes," JHEP 0611 (2006) 031 arXiv:hep-th/0607050. 
[45] M. Berg, M. Haack and B. Kors, "Loop corrections to volume moduli and inflation in string theory," Phys. Rev. D 71, 026005 (2005) [arXiv:hep-th/0404087].

[46] E. A. Bergshoeff, M. de Roo, S. F. Kerstan, T. Ortin and F. Riccioni, "SL(2,R)-invariant IIB brane actions," JHEP 0702 (2007) 007 arXiv:hep-th/0611036].

[47] A. Guarino and G. J. Weatherill, "Non-geometric flux vacua, S-duality and algebraic geometry," arXiv:0811.2190 [hep-th].

[48] J. Schon and M. Weidner, "Gauged N = 4 supergravities," JHEP 0605, 034 (2006) arXiv:hep-th/0602024.

[49] C. Bachas, "A Way to break supersymmetry," arXiv:hep-th/9503030; R. Blumenhagen, L. Goerlich, B. Kors and D. Lust, "Noncommutative compactifications of type I strings on tori with magnetic background flux," JHEP 0010, 006 (2000) arXiv:hep-th/0007024]; R. Blumenhagen, B. Kors and D. Lust, "Type I strings with F- and B-flux," JHEP 0102, 030 (2001) arXiv:hep-th/0012156.

[50] G. Villadoro and F. Zwirner, "D terms from D-branes, gauge invariance and moduli stabilization in flux compactifications," JHEP 0603 (2006) 087 arXiv:hep-th/0602120].

[51] O. Loaiza-Brito, "Freed-Witten anomaly in general flux compactification," Phys. Rev. D 76 (2007) 106015 arXiv:hep-th/0612088].

[52] G. Villadoro and F. Zwirner, "On general flux backgrounds with localized sources," JHEP 0711 (2007) 082 arXiv:0710.2551 [hep-th]].

[53] F. Marchesano, "D6-branes and torsion," JHEP 0605, 019 (2006) arXiv:hep-th/0603210].

[54] N. Kaloper and R. C. Myers, "The O(dd) story of massive supergravity," JHEP 9905, 010 (1999) arXiv:hep-th/9901045.

[55] M. Grana, R. Minasian, M. Petrini and A. Tomasiello, "A scan for new N=1 vacua on twisted tori," JHEP 0705 (2007) 031 arXiv:hep-th/0609124].

[56] A. Font, A. Guarino and J. M. Moreno, "Algebras and non-geometric flux vacua," arXiv:0809.3748 [hep-th]. 
[57] M. Grana, R. Minasian, M. Petrini and D. Waldram, "T-duality, Generalized Geometry and Non-Geometric Backgrounds," arXiv:0807.4527 [hep-th]; M. Grana, J. Louis and D. Waldram, "SU(3) x SU(3) compactification and mirror duals of magnetic fluxes," JHEP 0704, 101 (2007) arXiv:hep-th/0612237.

[58] C. M. Hull, "Generalised geometry for M-theory," JHEP 0707 (2007) 079 arXiv:hep-th/0701203]; P. P. Pacheco and D. Waldram, "M-theory, exceptional generalised geometry and superpotentials," JHEP 0809, 123 (2008) [arXiv:0804.1362 [hep-th]].

[59] G. Dibitetto, A. Guarino and D. Roest. Work in progress 